\title{
Local multiphase flow characteristics of a severe-service control valve
}

\author{
D. Singh ${ }^{\text {a }}$, A. M. Aliyu ${ }^{\text {a* }}$, M. Charlton ${ }^{\text {b }}$, R. Mishra ${ }^{\text {a }}$, T. Asim ${ }^{\text {c }}$, A. C. Oliveira ${ }^{a}$
}

${ }^{a}$ School of Computing and Engineering, University of Huddersfield, Queensgate, HD1 3DH, United Kingdom.

${ }^{b}$ Formerly of Trillium Flow Technologies, Britannia House, Huddersfield Road, Elland, West Yorkshire, HX5 9JR, United Kingdom.

'School of Engineering, Robert Gordon University, Garthdee Road, Aberdeen, AB10 7GJ, United Kingdom.

\section{Abstract}

For safety-critical industrial applications, severe-service valves are often used, and the conditions during operations can be either single phase or multiphase. The design requirements for valves handling multiphase flows can be very different to the single-phase flow and depend on the flow regime within valves. The variation in flow conditions during the operation of such valves can have a significant effect on performance, particularly in oil and gas applications where multiphase behaviour can rapidly change within the valve causing unwanted flow conditions. Current practices in designing and sizing such valves are based solely on global phase properties such as pressure drop of the bulk fluid across the valve and overall phase ratio. These do not take into account local flow conditions, as with multiphase fluids, the flow behaviour across the valve becomes more complex. In this work, wellvalidated computational fluid dynamics (CFD) tools were used to locally and globally quantify the performance characteristics of a severe service valve handling multiphase gas and liquid flow. Such flows are frequently encountered in process equipment found in vital energy industries e.g. process and oil \& gas. The CFD model was globally validated with benchmark experiments. Two valve opening positions of $60 \%$ and $100 \%$ were considered each with 5,10 , and $15 \%$ inlet air volume fractions to simulate real life conditions. The results show that while the non-uniformity in pressure field is along expected lines, there is severe non-uniformity in the local air, water and void fraction distributions within the valve trim. To quantify the phase non-uniformities observed, an equation for the distribution parameter was defined and used to calculate its value in each localised quarter within the trim. Phase velocity and void fraction data extracted from the CFD results were also used to obtain relationships for the local void fraction distribution and flow coefficient. The detailed investigation that has been carried out allows for local flow characteristics to be determined and embedded in sizing methodology for severe-service control valve systems with multiphase gas and liquid flow.

Keywords: Computational fluid dynamics, control valves, drift flux model, flow capacity, two-phase flow, valve trim.

\section{Introduction}

Severe service control valves are installed in pipelines where pressure differential requirements are very high and are used in a variety of industrial applications including those in the power generation, chemical, and oil \& gas industries. These valves are carefully designed to ensure controlled heat and mass transfer performance in several critical service applications. The design methodology for these valves are carefully developed and owing to lack of information about the local flow field within the valve internal parts (e.g. its trim), design methodologies mostly rely on global performance indicators and variables. Several investigations have been carried out in understanding local flow features within the valves and linking the design methodologies with local flow features to avoid problems such as cavitation and flashing [1]-[4]. Kang et al [1] quantified the effects of fittings on valve's global flow capacity. Lin and Schohl [4], carried out a numerical investigation on a butterfly valve and established 
its local flow characteristics without establishing design criteria for such valves. Similarly, Yang et al [2] investigated the effect of complex flow field on vibration characteristics without establishing detailed performance of the valve. An et al [5] focussed more on overall flow capacity change with valve opening position (VOP) without understanding its effect on the local flow field and hence on local flow capacities at important sections. Lisowski et al. [6]-[8] carried out detailed investigation on the flow characteristics of a proportional flow control valve. The authors reported a good agreement between the numerical predictions and the experimental data. Computational fluid dynamics (CFD) was then used to develop and test new design features in the valves. Asim et al. [9] developed a novel methodology to correlate local flow capacity within the trim with the specific flow path and with disc location inside the trim. This allows designers to incorporate these local quantities in design methodologies and thus attempt to eliminate the possibility of cavitation within the complex flow field. In order to investigate flow path geometry on the local flow capacity within the trim at various valve openings, Asim et al. [10] [11] conducted comprehensive CFD studies on a valve system. Cylinder shape modification within the valve trim was found to have significant effects on energy losses in the trim. These works however are only focussed on single-phase water flow and there are very few studies on gas-liquid multiphase flow through control valves. Furthermore, the few available works in the literature for multiphase flow in control valves largely analyse global parameters associated with the system. Control valve sizing is based on the BS standard 60534-2-1 [12], which involves calculating the flow coefficient $K_{v}$ required for a certain fluid flow rate and pressure drop across the valve. The standard also covers compressible, turbulent and laminar and choked single-phase fluid flows. However, for multiphase flowing media, several different approaches are being used each with certain inaccuracies and there is not yet a standardised approach to the process [13].

Nevertheless, a very small number of authors have reported works on multiphase flow characteristics in control or relief valves. Typical of the few works concerning relief valves are those of Dempster and Alshaikh [14], [15]. They experimentally and numerically investigated the flow characteristics within a safety valve under multiphase flow conditions with widely varying operating parameters. In both studies, they obtained the effects of inlet conditions on flow characteristics and overall flow capacity of the valve, as well as the forces on the disc in the safety valve. They did not carry out any detailed study on the local velocity or pressure characteristics or flow capacity within the valve, or how these can be related to the valve coefficient $K_{v}$ and used for sizing of the valve.

In contrast, for control valves, there have been more studies in the literature including a number of attempts to include the effect of gas fraction in determining $K_{v}$ when gas-liquid mixtures flow through the valve. Because gas compressibility (or expansion) is affected by the gas void fraction or vapour quality, the inlet flow regime and the total inlet flow rate is dependent on gas fraction or compressibility which is not the case in single-phase liquid flow. In such cases, the overall density of the mixture at the control valve inlet depends on the gas void fraction. The equation used for calculating a control valve's flow capacity for non-choked, incompressible, single-phase fluid flow is given as [9], [16]:

$$
K_{v_{\text {valve }}}=11.56 Q \sqrt{\frac{\rho / \rho_{o}}{\Delta P}}
$$

where $\Delta P$ and $Q$ are expressed in the units of $\mathrm{kPa}$ and $\mathrm{m}^{3} / \mathrm{h}$ respectively, and the constant 11.56 is used only with these units [12]; $\rho$ and $\rho_{o}$ are the densities of the flowing liquid and water at ambient temperature respectively, meaning the density ratio ${ }^{\rho} / \rho_{o}$ is the specific gravity of the liquid and is 1 if water is the liquid concerned. In the case of multiphase flow, a few attempts have been proposed to calculate the flow coefficient of control valves taking account of gas flowing with the liquid. It was 
noted that the simplest of these models is known as the "addition" [17], [18] or "sum of CVs" model. This model treats each phase separately whereby the flow coefficient of each phase is calculated. These are then added to give an overall flow coefficient, but it was observed that this model tends to underestimate the flow coefficient [18]. Sheldon and Schuder [19] proposed a method that attempted to correct this, which makes use of a correction factor that depends on the phase volume fractions at the valve inlet. Diener et al. [18] noted that a calculation scheme can be used that arises from the simplifying assumption that the flowing gas and liquid mixture is well mixed or homogenous, and travel at the same velocity (with no phase slippage) i.e. $\rho_{\text {mix }}=\alpha_{\text {inlet }} \rho_{\text {air }}+\left(1-\alpha_{\text {inlet }}\right) \rho_{\text {water }}$. The terms $\rho_{\text {air }}, \rho_{\text {water }}, \rho_{\text {mix }}$ and $\alpha_{\text {inlet }}$ are the air, water, two-phase mixture densities and inlet air volume fraction respectively. As such, Equation (1) for two-phase gas-liquid flow can be written as:

$$
K_{v_{\text {valve }}}=11.56 Q_{\text {mix }} \sqrt{\frac{\rho_{\text {mix }} / \rho_{o}}{\Delta P}}
$$

The mixture density is then given as the reciprocal of the mean specific volume of the two phases at the operating temperature and pressure, and it is then used in Equation (1). Diener et al. [13], [18], [20] however mentioned that that these methods can give estimates of the valve flow coefficient that can deviate from experimental measurements at higher volume flow rates, as the effect of inter-phase slip becomes more pronounced. They proposed a method that accounts for the effect of gas compressibility when calculating the valve flow coefficient $K_{v_{v a l v e}}$ by introducing a "multiphase expansion factor" $\left(1 / Y_{M P}\right)$ to the right-hand side of Equation (1):

$$
K_{v_{\text {valve }}}=\frac{11.56 Q}{\sqrt{\Delta P}} \frac{1}{Y_{M P}}
$$

For a single-phase liquid, $Y_{M P}=Y=1$ and Equation (3) simplifies to Equation (1). The calculation steps for $Y_{M P}$ are given in their articles [13], [18]. Their method is explicit and extends previous methods namely the $\omega$-method and the homogenous non-equilibrium-Diener/Schmidt (or HNE-DS) method. The $\omega$-method developed by Leung [21], [22] uses the fluids' physical properties (densities of the two phases, their specific heat capacities as well as the liquid vaporisation enthalpy). It however tends to underpredict the mass flow rate and hence $K_{v_{v a l v e}}$ [20]. The homogenous non-equilibriumDiener/Schmidt or HNE-DS method Diener [23] is implicit and implemented via an iterative procedure. We note that the explicit multiphase expansion factor $\left(1 / Y_{M P}\right)$ method though not iterative, is mainly applicable to non-equilibrium flows that contain evaporating and condensing vapours (e.g. steam/water) at high pressures of up to $10 \mathrm{MPa}$. Furthermore, it requires state variables and properties as input parameters (i.e. enthalpies, specific heats for both fluids at the prevailing conditions of temperature and pressure) as well as thermodynamic equations and correlations in order to account for the inlet gas volume fraction in Equation (3). Along with these drawbacks, this and similar procedures ultimately only give the global two-phase flow parameter across the valve and do not give any information on their dependence on local flow, phase distribution and other parameters within the valve and trim.

From the foregoing discussion, it can be summarised that control valve design and sizing with multiphase flowing mixtures has largely been based on global flow parameters e.g. pressure drop across the entire valve system for a given mixture flow rate. The works that deal with local parameters within the valve and its trim are few [9]-[11] and even so, they only apply to single-phase liquid flowing through the valve. One reason behind this apparent weakness in existing methods is limited knowledge about the flow field with two flowing phases and their distribution through complex geometries. 
In this study, local phase flow fields as well as global and local valve flow coefficient was determined under multiphase air-water conditions using a well-validated CFD model. The effect of inlet gas volume fraction on local pressure, void fraction, and phase velocity distributions within the valve trim were also thoroughly investigated. Local mixture volumetric flow rates, mixture densities, and velocities were extracted at several important locations within the trim from the CFD results and the local flow capacities at respective rows in different disc quarters located at the top, middle, and bottom locations have been obtained. We then derive several relationships including for $K_{v_{\text {row }}}$ as a function of local gas void fractions and their localised position inside the trim. It is hoped that the correlations developed in this work can be used alongside current sizing methods to improve local performance compliance.

\section{CFD modelling of the control valve and experimental validation}

\subsection{CFD modelling}

The numerical modelling of the control valve and the multi-stage continuous-resistance trim was performed using the Ansys 19.2 software to determine the relationship between the local flow behaviour and the global performance indicators with a two-phase gas-liquid mixture flowing within the system. As with any CFD-based numerical modelling, the current modelling is composed of the pre-processing, solver setup and post-processing stages. The pre-processing stage is further subdivided into flow domain geometry creation/meshing; solver setup, which includes the specification of boundary conditions, material properties, turbulence modelling, convergence criteria and initial solution. These all require special consideration when performing simulations of multiphase flows through complex geometries. The post-processing stage involves the analysis of results obtained in order to display flow features within the complex geometry. Furthermore, the two-dimensional sectional planes to be considered and flow variables to be investigated need to be carefully selected.

\subsubsection{Control valve and trim geometry}

The geometry of the control valve and connecting horizontal pipes is as shown in Figure 1(a). The specific model of control valve used in this study was manufactured by an industrial collaborator, it is widely installed and used in the process industry. As the figure shows, the length of the inlet and outlet pipes are 2D and 6D respectively which is in accordance with the BS EN Standards [12], [16], [24]. The internal diameter $D$ of the inlet and outlet pipes is the same and the exit section is longer because there is need to allow for reasonably enough flow development or stabilisation after the fluid exits the valve before pressure and other measurements are taken. Figure 1 (b) shows the inlets for air and water. Air enters through the circular area in the centre of the pipe and water enters through the annular area around the air inlet. Figure 1 (c) gives the geometry of the trim showing the stack of discs and external row of cylinders forming the obstruction to flow and giving rise to the flow paths. Internally, there are five such rows in staggered formation as we move inwards from the periphery to the centre of the trim. This is shown in Figure 1 (c) with $d_{i}$ being the diameter of all the cylinders in row $i$. As such, the diameter of all cylinders in the outer or first row is $d_{1}$; and $d_{2}$ denoting the diameter of those in the second row and so on, such that $d_{1}>d_{2}>d_{3}>d_{4}>d_{5}$. The flow direction within the discs is from the outer diameter towards the inner diameter, i.e., from row 1 to row 5 as shown in Figure 1(d). Row 1, 3 and 5 have 7 flow paths whereas rows 2 and 4 have 8 flow paths. Severe-service control valves have complex flow paths and it is important to understand the flow characteristics through the intricate pathways so as to eliminate unwanted effects such as noise, vibrations, and cavitation. Since the cylinders are staggered, there are different number of flow paths in different rows. The cylinders are staggered so that there is a staged reduction in pressure. Hence, it is expected that the number of flow paths for different rows will exhibit different flow behaviour and these need 
to be understood and quantified. Accordingly, the centre of each row of cylinders forms a radius to the centre of the trim and this is denoted as $r_{i}$ such that $r_{1}>r_{2}>r_{3}>r_{4}>r_{5}$. Figure 1 (e) shows the location of planes $A-F$ such that plane $A$ is at the entrance of row 1 , plane $B$ is at the exit of Row 1 and at the entrance of Row 2, etc. At each of these planes, data were extracted for post-processing and further analysis.

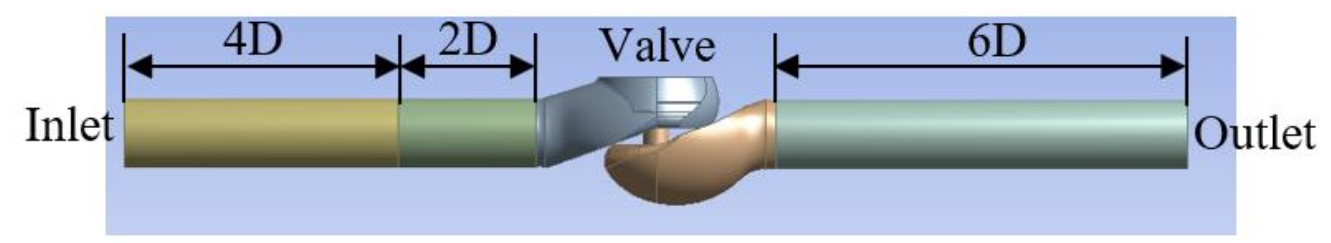

(a)

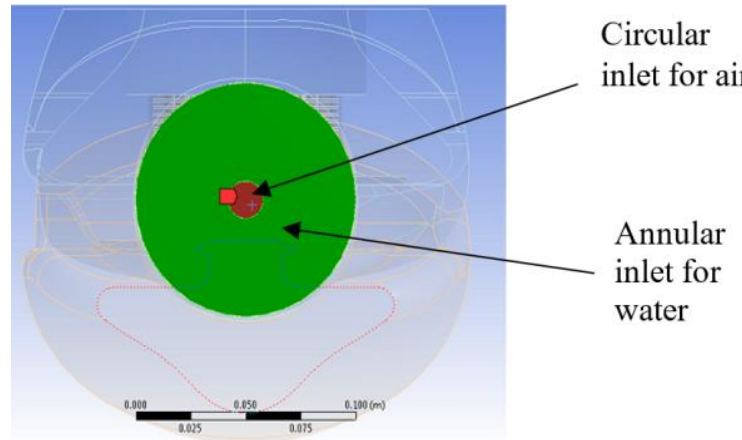

(b)

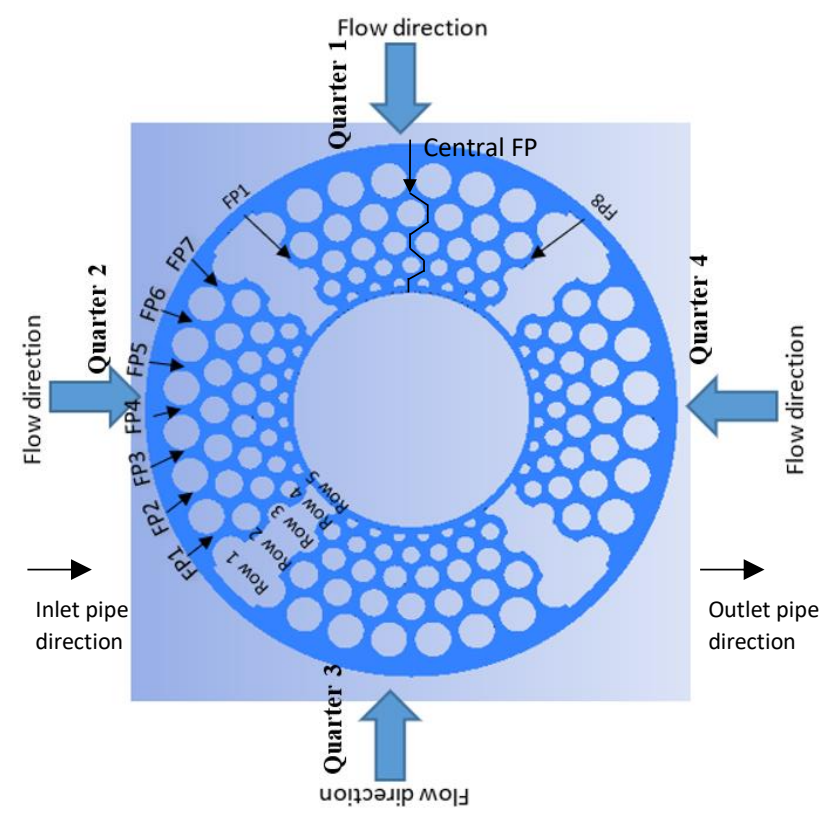

(d)

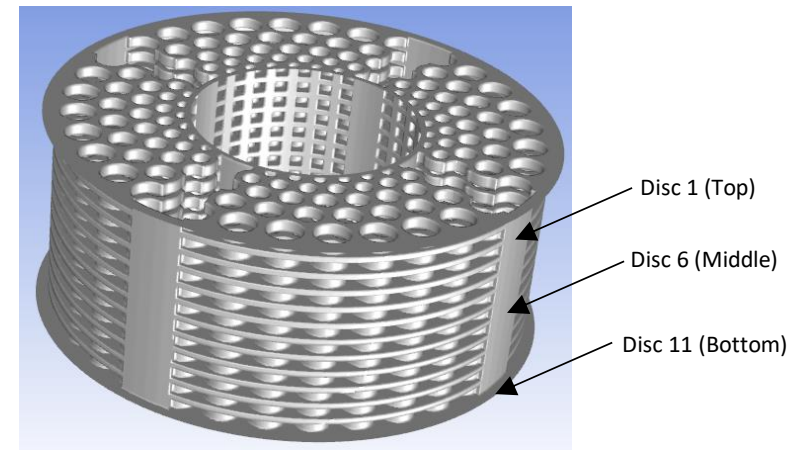

(c)

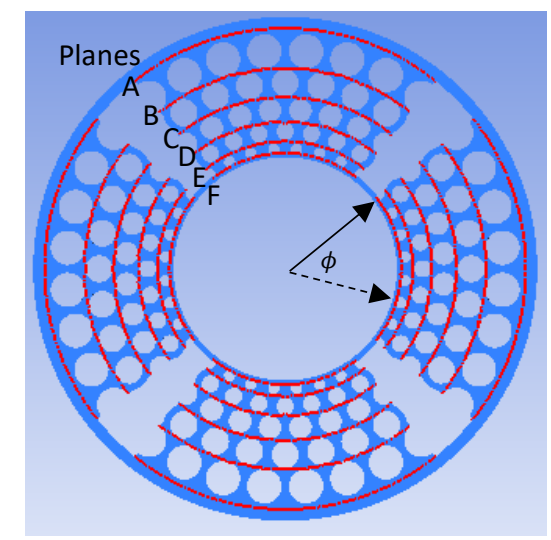

(e)

Figure 1: CAD model of (a) the control valve/inlet and outlet pipe geometries (b) water and air inlets (c) the valve trim geometry and disc numbering (d) details of a disc within the trim - FP denotes "flow path" (e) location of planes in a trim disc for data extraction and analysis

\subsubsection{Flow domain meshing}

Appropriately meshing the flow domain is a vital step in CFD modelling because it guarantees the discretisation of the flow domain into smaller parts known as mesh elements. The size of mesh elements impacts on the accuracy of flow near areas with high velocity gradients [11]. A large number of mesh elements in the order of millions (for 3-dimensional simulations) is required to accurately 
predict local flow within the flow paths, and the mesh elements become finer at regions with strong curvature/flow gradients and especially around cylinders with smaller diameter [25], [26] such as those around Row 5 of the trim. Figure 2 (a) and (b) give an appreciation of this and depict that two types of mesh elements were combined in the flow domain. These are hexahedral and tetrahedral mesh elements. Hexahedral mesh elements were utilised in regions of the mesh where the geometry is fairly uncomplicated and symmetrical, such as the inlet and outlet pipes. On the other hand, tetrahedral elements were used in regions with more complicated geometry as is clearly the case within the valve and trim. The type of mesh element used in an area is additionally decided by flow symmetry and it is widely published and agreed that hexahedral mesh elements should be used for symmetric flows, while tetrahedral elements should be used for asymmetric flows as they provide results that are more accurate [27]. To capture near-wall effects in between the rows of the valve trim that could be different from other rows, a more rigorous control was needed to generate the mesh within these small flow areas. As a result, a proximity and curvature technique based on the flow domain walls i.e. around the cylinders was used to achieve the required mesh elements within the trim [28]. Further ensuring the accurate prediction of the flow parameters in the near-wall regions, especially around trim's cylinders; two additional layers of hexahedral elements per cylinder were created with growth rate of $20 \%$. A close look at Figure 2 (b) reveals these near-wall mesh layers.

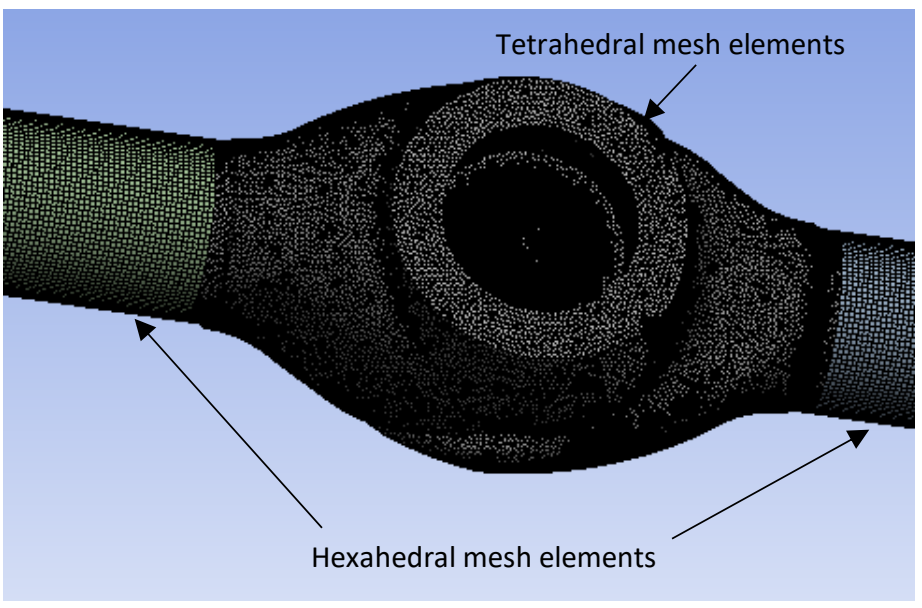

(a)

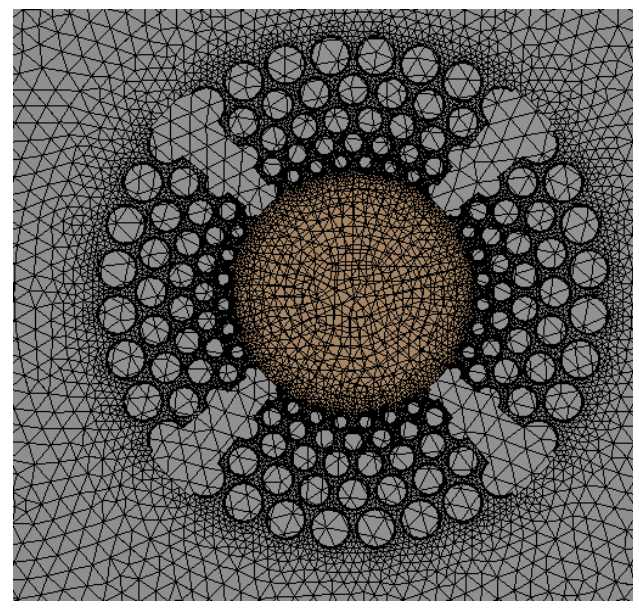

(b)

Figure 2: Mesh of (a) the valve flow domain (b) the trim

A mesh independency study was conducted using meshes with 5.1 million, 5.4 million and 6.1 million elements. The volume fraction was monitored at the outlet and when the flow passes through the valve seat. The results are shown in Table 1 with the percentage difference is shown in brackets. As shown, there is very small difference (less than $\pm 5 \%$ in most cases) between the outlet volume fractions for all the different mesh sizes. For example, outlet volume fraction changes by $4.8,-6.4$, and $1.5 \%$ for 5,10 , and $15 \%$ respectively as the number of mesh elements increases from 5.4 to 6.1 million. Hence for computational efficiency, the mesh with 5.4 million elements was used for the simulation presented henceforth in this work.

Table 1: Mesh independence study - volume fraction results

\begin{tabular}{cccc}
\hline $\begin{array}{c}\text { Air volume } \\
\text { fraction - inlet }\end{array}$ & $\begin{array}{c}\text { Mesh elements } \\
\text { (million) }\end{array}$ & $\begin{array}{c}\text { Air volume fraction - } \\
\text { Seat }\end{array}$ & $\begin{array}{c}\text { Air volume fraction } \\
\text { - Outlet }\end{array}$ \\
\hline \multirow{2}{*}{0.05} & 5.1 & 0.0471 & 0.0535 \\
& 5.4 & $0.0458(-2.8 \%)$ & $0.0510(-4.7 \%)$ \\
0.1 & 6.1 & $0.0478(+4.4 \%)$ & $0.0538(+4.8 \%)$ \\
\hline 0.1 & 5.1 & 0.0878 & 0.1105
\end{tabular}




\begin{tabular}{cccc} 
& 5.4 & $0.0903(+2.9 \%)$ & $0.1066(-3.5 \%)$ \\
& 6.1 & $0.0895(-0.86 \%)$ & $0.0998(-6.4 \%)$ \\
\hline \multirow{3}{*}{0.15} & 5.1 & 0.1340 & 0.1530 \\
& 5.4 & $0.1271(-5.2 \%)$ & $0.1564(+2.2 \%)$ \\
& 6.1 & $0.1331(+4.8 \%)$ & $0.1587(+1.5 \%)$ \\
\hline
\end{tabular}

\subsubsection{Turbulence modelling}

In modelling flows using the Reynolds-Averaged Navier-Stokes (RANS) approach, the use of an appropriate turbulence model for simulating complex-geometry flows requires special consideration. This is more so in the case of multiphase flows. Various turbulence models have been discussed in reference texts [27]-[30]. These include the Reynolds Stress, $k-\varepsilon$, and the $k-\omega$ turbulence models, with each model more suited to a specific application. Here, the two-equation $k-\omega$ Shear Stress Transport (SST) model was selected for turbulence modelling. The main reason being that the $k-\omega$ SST model has long been shown to be superior in appropriately modelling flows with severe velocity gradients [31]. In this study, these adverse velocity gradients are expected to arise within the trims due to the complex path changes that the flow is expected to encounter. Moreover, it has been shown that the $k-\omega$ model should be preferably selected for internal flows [32]. The $k-\omega$ SST model contains a blending function for dealing with near-wall effects and it additionally has the definition of the turbulent viscosity modified to account for turbulent shear stress transport. All these are generally regarded to make the k- $\omega$ SST model reliable and widely applicable to a wide range of flows [27], [28]. In addition to selecting the $k-\omega$ SST model, mesh refinements were made. Mainly, mesh layers were strategically placed at locations in the far field of the walls. These locations were selected as the $k-\omega$ SST turbulence model models the viscous sub-layer. Nevertheless, $k-\omega$ SST model resolves the flow in the log-law region and as a result the mesh layers were densely packed in the log-law region. Summarily, in addition to the three-dimensional RANS equations, the continuity or mass conservation equation, the $k-\omega$ equations were also solved iteratively. A comparative investigation between SST-k- $\omega$ and standard $k-\varepsilon$ turbulence model performed by Asim et al. [11] showed that the k- $\omega$ SST model underpredicts the static gauge pressure at the inlet pipe of the current control valve's flow domain by more than $5 \%$. Conversely, the extensively applied standard $k-\varepsilon$ model overpredicted the same inlet static gauge pressure with a difference of nearly $19 \%$.

\subsubsection{Solver setup}

In multiphase simulations, mixture multiphase model has been used where water is modelled as the primary phase and air as the secondary phase. Boundary conditions at the inlet for different air volume fractions are shown in Table 2. Based on the experimental condition that gives the required 5, 10, and $15 \%$ gas volume fraction, the air inlet velocity was calculated for a specific liquid inlet velocity and volume fraction. To achieve this, the air velocity was calculated from the definition of the air volume fraction $\left(\alpha=Q a /\left(Q_{a}+Q_{l}\right)\right)$. Neglecting inter-phase slip, the gas inlet velocity is calculated using this relationship as follows:

$$
v_{g}=\frac{\alpha_{\text {inlet }} A_{l} v_{l}}{\left(1-\alpha_{\text {inlet }}\right) A_{g}}
$$

where $\alpha_{\text {inlet }}$ is the air inlet volume fraction; $v_{g}$ and $v_{l}$ are the gas and liquid velocities respectively; and $A_{g}$ and $A_{l}$ are the gas and liquid inlet pipe cross-sectional areas respectively. The outlet boundary condition was set to atmospheric pressure. The walls were modelled with no-slip condition. For single phase simulations, the inlet gauge pressure was defined as $342.84 \mathrm{kPa}$ similar to the experiments. In solver settings, the SIMPLE pressure-velocity coupling scheme was used for single phase whereas for 
multiphase simulations the coupled scheme was used. Convergence was monitored using mass flow rates of both air and water at the outlet and volume fraction of air at the outlet. The simulations were carried out at steady state conditions.

Table 2: Inlet boundary conditions

\begin{tabular}{ccc}
\hline $\begin{array}{c}\text { Volume } \\
\text { fraction (\%) }\end{array}$ & $\begin{array}{c}\text { Water inlet } \\
\text { velocity }(\mathrm{m} / \mathrm{s})\end{array}$ & $\begin{array}{c}\text { Air inlet } \\
\text { velocity }(\mathrm{m} / \mathrm{s})\end{array}$ \\
\hline 5 & 1.22 & 2.5 \\
10 & 1.22 & 5.3 \\
15 & 1.22 & 8.4 \\
\hline
\end{tabular}

\section{$5 \quad 2.2$ Benchmarking experiments}

6 Validation of numerical models is of great importance for the validity of the numerical results. In order 7 to benchmark and hence validate the numerical results obtained in this study, experiments were 8 carried out using the same control valve and trim. This allowed the determination of the global flow 9 capacity of the control valve $K_{v_{v} \text { alve }}$ from which $K_{v_{\text {trim }}}$ was calculated. A flow loop was specially designed and built for this purpose and a schematic is given in Figure 3. The loop consists of the severeservice diaphragm-type control valve, which has two horizontal pipes, of internal diameter of $100 \mathrm{~mm}$, connected to its up- and downstream ends. The upstream end was connected to a $24.1 \mathrm{~kW}$ centrifugal pump which draws water from a $1-\mathrm{m}^{3}$ plastic reservoir tank via a Polyvinyl Chloride (PVC) pipe. The pump has the capacity to deliver a head of up to $55 \mathrm{~m}$ and a maximum flow rate of $93.6 \pm 1 \% \mathrm{~m}^{3} / \mathrm{h}$. For metering the water flow, use was made of a turbine flow meter that measures up between 1.8 and $120 \mathrm{~m}^{3} / \mathrm{h}$ with a small $0.2 \%$ pressure drop across it at the maximum flow rate.

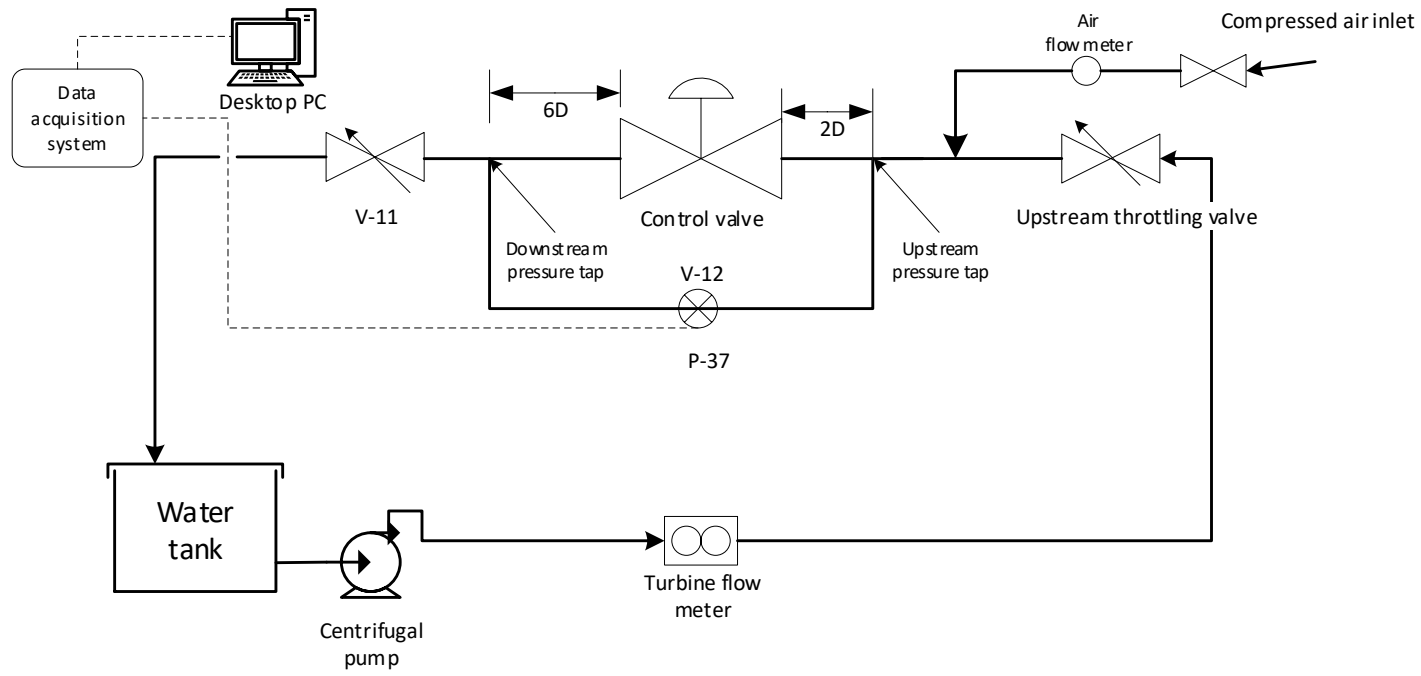

(a)

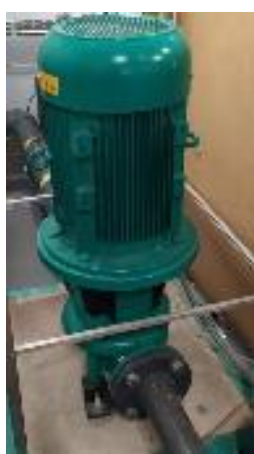

(b)

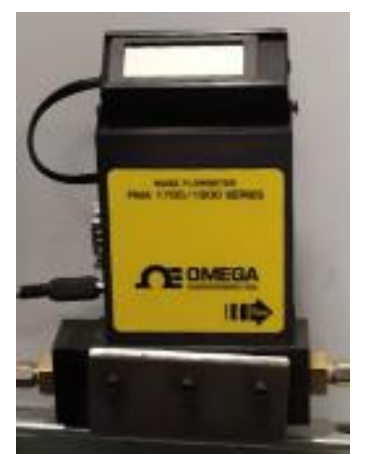

(c)

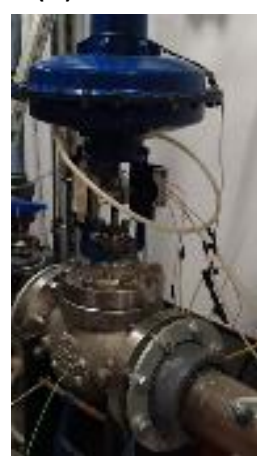

(d)

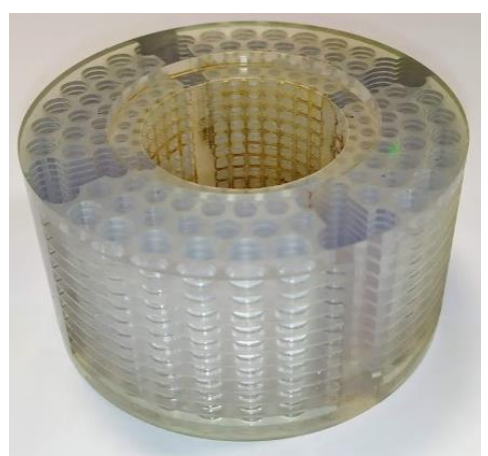

(e) 
Figure 3: Experimental facility showing (a) schematic of flow loop with details of test section and dimensions of up/downstream sections (b) centrifugal pump (c) air mass flow meter (d) control valve (e) control valve trim

To determine the pressure loss across the valve, tappings were made at locations of $2 \mathrm{D}$ before and $6 \mathrm{D}$ after the valve as per BS EN 60534-2-5 [24]. Four pressure tappings were installed at each of the positions to measure the average pressure within the pipeline rather than using one which gives a point pressure and may not be representative of the entire pipe section. The pressure tappings were connected to a differential pressure transducer (of $-1-10$ barg $\pm 0.25 \%$ measuring range) and the pressure drop across the control valve was determined for each set of operating conditions. Furthermore, Table 3 shows a comparison of the valve flow coefficient calculated by CFD and experimentally for single-phase water flow as well as for 5 and $10 \%$ air inlet volume fraction. Error propagation analysis was carried out to determine the uncertainty in experimental $K_{v_{v} a l v e}$ which is given by [ref]:

$$
\varepsilon_{K_{v_{\text {valve }}}}=\sqrt{\left(\varepsilon_{Q_{\text {mix }}} \frac{\partial K_{v_{\text {valve }}}}{\partial Q}\right)^{2}+\left(\varepsilon_{\Delta P} \frac{\partial K_{v_{\text {valve }}}}{\partial \Delta P}\right)^{2}}
$$

where $\varepsilon$ is the uncertainty in the quantity in subscript; $\partial K_{v_{v a l v e}} / \partial Q$ and $\partial K_{v_{v a l v e}} / \partial \Delta P$ are the partial derivatives of $K_{v_{\text {valve }}}$ obtained by differentiating Equation (2). The uncertainties $\varepsilon_{Q_{\text {mix }}}$ and $\varepsilon_{\Delta P}$ in $Q$ and $\Delta P$ are those of the flow meter and differential pressure transducer respectively and whose values have been given in the preceding paragraph. After calculating the absolute error with Equation (5), the relative percentage error: $\frac{\varepsilon_{K_{v_{v a l v e}}}}{K_{v_{\text {valve }}}} \times 100 \%$ is calculated and yields $0.55-0.57 \%$ experimental uncertainty in $K_{v_{\text {valve }}}$ for $0-10 \%$ inlet air volume fractions respectively. For the $K_{v_{\text {valve }}}$ values proper, a comparison between experiments and CFD results in percentage absolute differences of between 3.2 and $6.9 \%$ (Table 3 ). These values are acceptable and essentially validate the CFD model since random errors can occur during any of the experimental runs as well as geometrical imperfections within the trim which are very difficult to capture in the modelling. Furthermore, it was noted that numerical convergence can also contribute to the observed discrepancies between the CFD and experimental values.

Table 3: Numerical and experimental $K_{v_{v a l v e}}$ at $100 \%$ valve opening position for CFD validation

\begin{tabular}{cccc}
\hline \multirow{2}{*}{ Inlet air volume fraction (\%) } & \multicolumn{2}{c}{$\boldsymbol{K}_{\boldsymbol{v}_{\text {valve }}}$} & \multirow{2}{*}{ \% Absolute difference } \\
\cline { 2 - 4 } & Experimental & CFD & 3.27 \\
5 & $37.52 \pm 0.57 \%$ & 36.29 & 6.86 \\
10 & $35.49 \pm 0.55 \%$ & 33.06 & 5.87 \\
\hline
\end{tabular}

\section{Results and discussion}

\subsection{Flow within trim with single-phase water}

The valve trim used in this study is geometrically complex and thus results in complex pressure and velocity fields. To establish the effect of complex geometrical pattern on the resulting flow field, a detailed investigation of the local pressure and velocity characteristics within the flow paths will be carried out. In this section the flow characteristics corresponding to single-phase flows have been explained so that the multiphase flow results can be benchmarked against these results. The top half of Figure 4 shows flow field corresponding to the top, middle and bottom discs within the existing trim. It shows that the pressure field is uniform, and the gradients exist in the flow direction (radial). 
The inlet pressure for the middle and bottom discs are like that at the top, but it is the outlet pressures where marked differences occur. As a result, more significant pressure drops occur at the bottom disc than observed at the top.
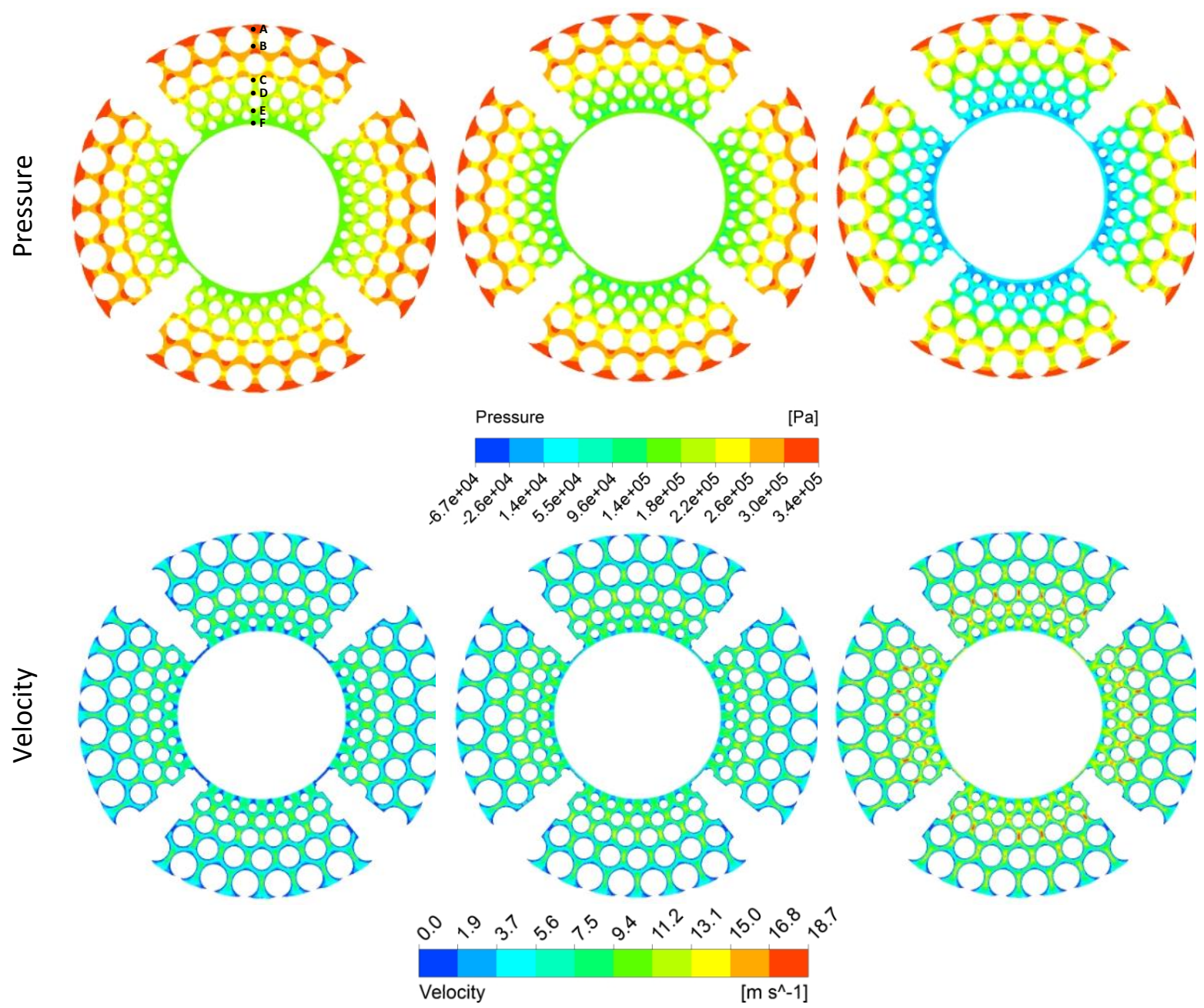

(a)

(b)

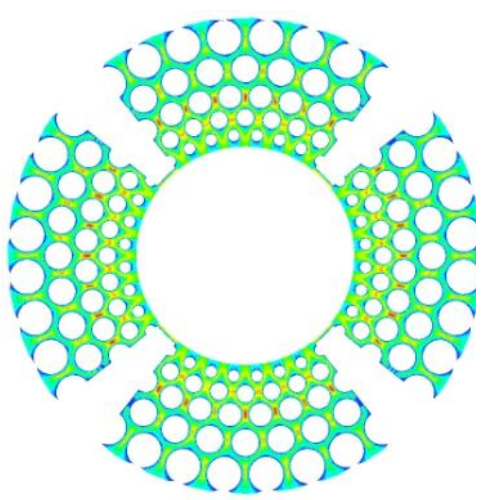

(c) Figure 4: Single-phase pressure and velocity fields corresponding to the (a) top, (b) middle and (c) bottom
disc at an average inlet flow velocity of $10 \mathrm{~m} / \mathrm{s}$

The corresponding velocity field (bottom half of Figure 4) can also be seen to be highly complex. The flow velocities change substantially when the fluid flows through the narrow pass formed between the cylinders. However, the flow velocity magnitude is highest in the flow paths of the 3rd row, compared to flow paths of other rows. Again, the velocity gradients exist predominantly in the flow direction because of area change. This also brings about non-uniform velocity gradients in the circumferential direction. The cylinder dimensions and their arrangement provide a variable resistance to the flow and the total value of flow capacity of trim is dictated primarily by the arrangement of the cylinders. For single phase flow, the primary concern is the geometry selection which should avoid cavitation/flashing and velocities should be such that the erosion potential of the trim can be minimised. The maximum velocity is about $18.5 \mathrm{~m} / \mathrm{s}$ within the bottom disc which can be considered to be in the normal conditions of operation. The velocity field within the top disc has been depicted in Figure 5 . In order to quantitatively analyse the flow velocity magnitude within the different flow paths of the trim, normalised velocity profiles have been drawn in each flow path. The ratio of maximum velocity to average velocity changes substantially along the flow path. The geometric details of these flow paths covered by a quarter of the disc indicate that $77^{\circ}$ of the whole quarter is in the flow domain. Figures $8(a-c)$ depict normalised flow velocity magnitude profiles within the flow paths 
for the top, middle and bottom discs respectively. There are different numbers of flow paths in different rows (rows 1, 3 and 5 have 7 flow paths, while rows 2 and 4 have 8 flow paths each). Furthermore, the flow velocity magnitude has been normalised with the maximum flow velocity within the trim. It shows that the velocity profile corresponding to row 3 is the highest (represented by the blue dashed line) giving a $u / u_{\max }$ ratio of approximately 1 . Those in rows 2 and 4 give ratios of about 0.75 to 0.8 of the maximum obtained at row 3. Asim et al. [9] have shown that the effective flow area for this trim at row 3 is less than that in rows 2 and 4, and hence, the flow velocity in the locality of row 3 will be higher, which can result in higher hydrodynamic losses. Furthermore, row 5 has the lowest velocity and whereas row 1 has velocity profile of intermediate magnitude to those of rows 3 and 5. Row 2 profile has higher local velocity as compared to row 4 . All the above information corresponds to $100 \%$ valve opening. The flow through discs is likely to be affected by the valve opening position as it is expected that velocity and pressure fields will change as different number of discs are in operation.

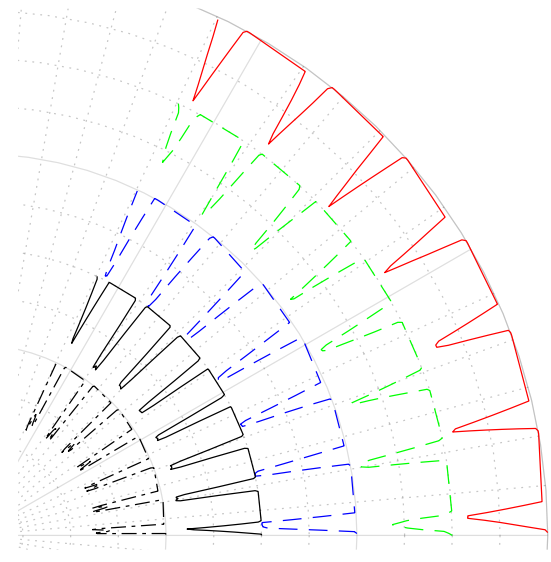

(a)

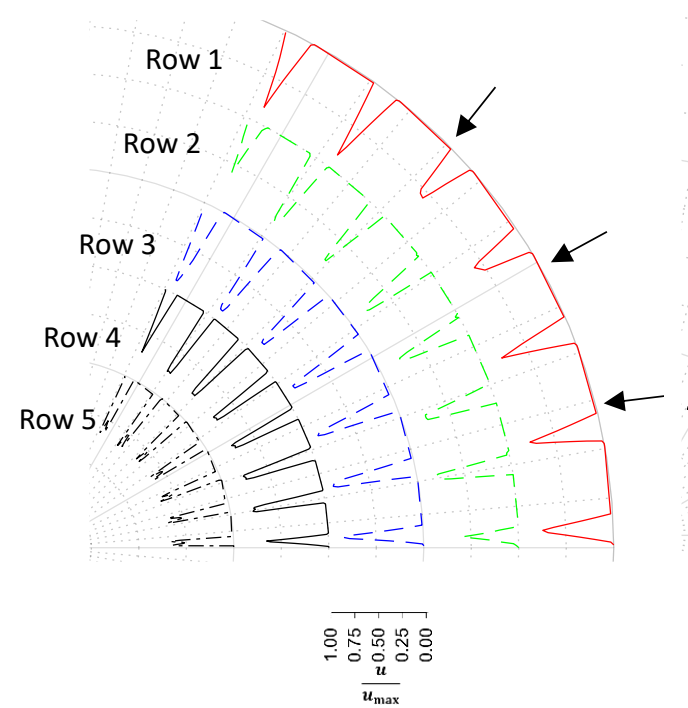

(b)

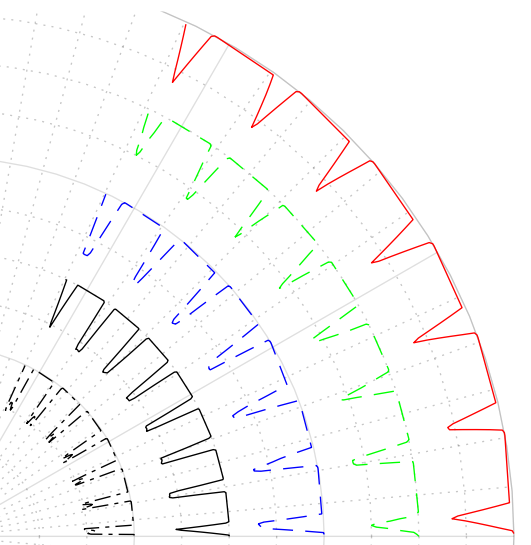

(c)

--- $100 \%$ VOP

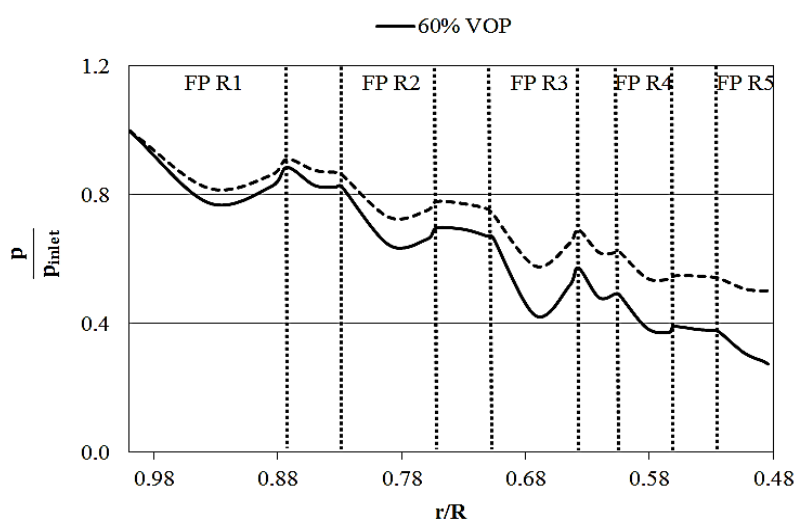

(d)

Figure 5: Non-dimensional single-phase water velocity profiles across different flow paths in a quadrant of the (a) top - Disc 1 (b) middle - Disc 6, and (c) bottom - Disc 11 at 100\% VOP for rows 1-5. The $u / \boldsymbol{u}_{\text {max }}$ axis applies to all rows, and arrows indicate direction of flow. (d) Variation of single-phase pressure at different valve opening positions for the central flow path in the first quadrant

To quantify this effect, pressure variation in the top disc has been evaluated for $100 \%$ valve opening position as well as $60 \%$ valve opening position. Pressure drop is higher in the case of $60 \%$ valve opening condition because of higher flow velocities for the same inlet mass flow rate as the area available to flow has decreased. The foregoing results and discussion have established that the relationship 
between trim/flow path geometry and the flow field is a complex one. It is hence imperative to quantify these effects for multiphase conditions because most of the multiphase design for such trims are based on very simplified assumptions. These assumptions need to be tested at both local and global level based on the flow field characteristics obtained.

\section{$5 \quad 3.2 \quad$ Flow within the trim under gas-liquid multiphase conditions}

6 To observe and evaluate multiphase effects, simulations were carried out for air-water mixture at

7 three air volume fraction values of $5 \%, 10 \%$ and $15 \%$ and the flow field was evaluated.

Vol.

fraction

Top disc

Middle disc

Bottom disc

[\%]

5
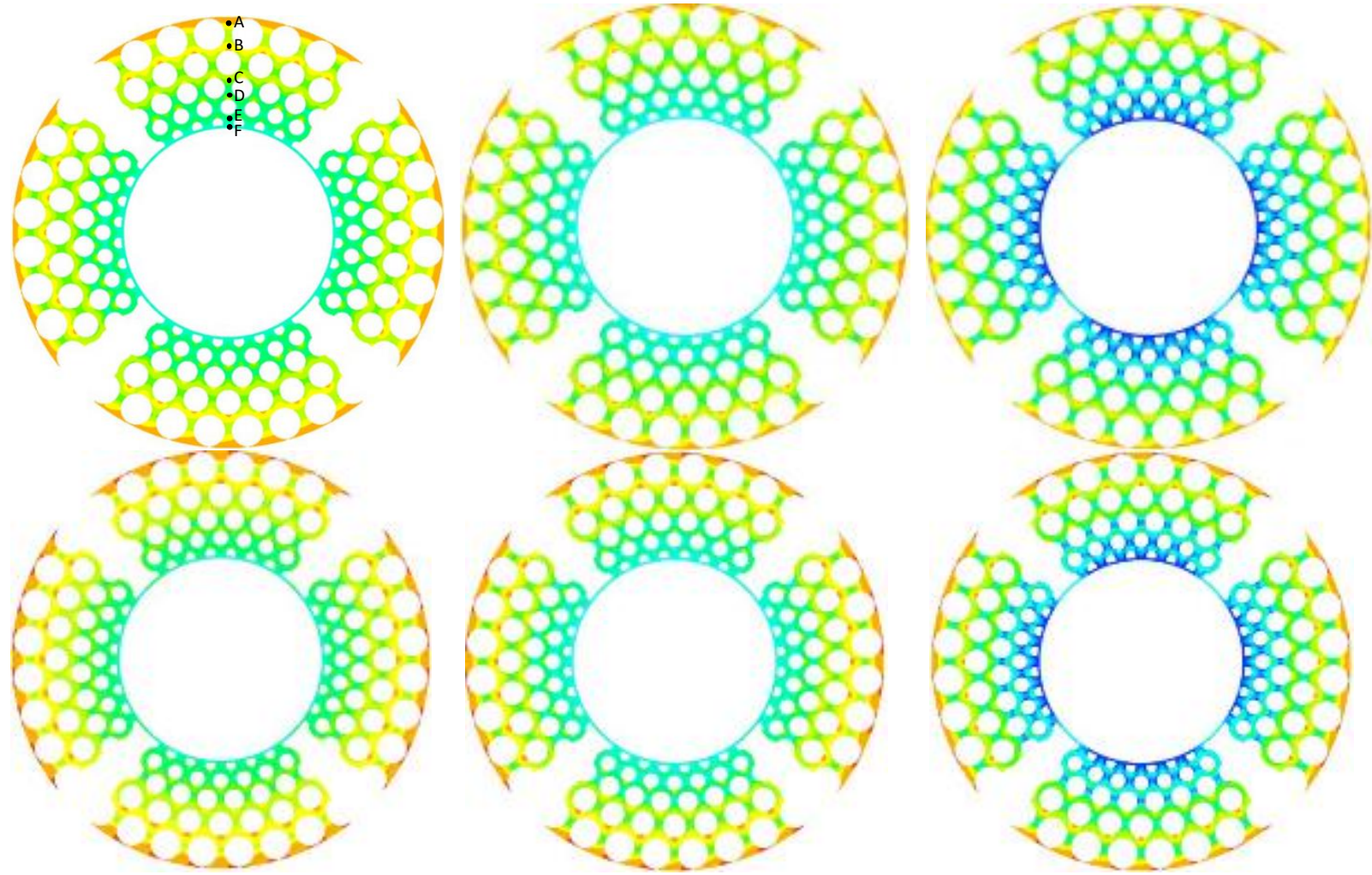

10
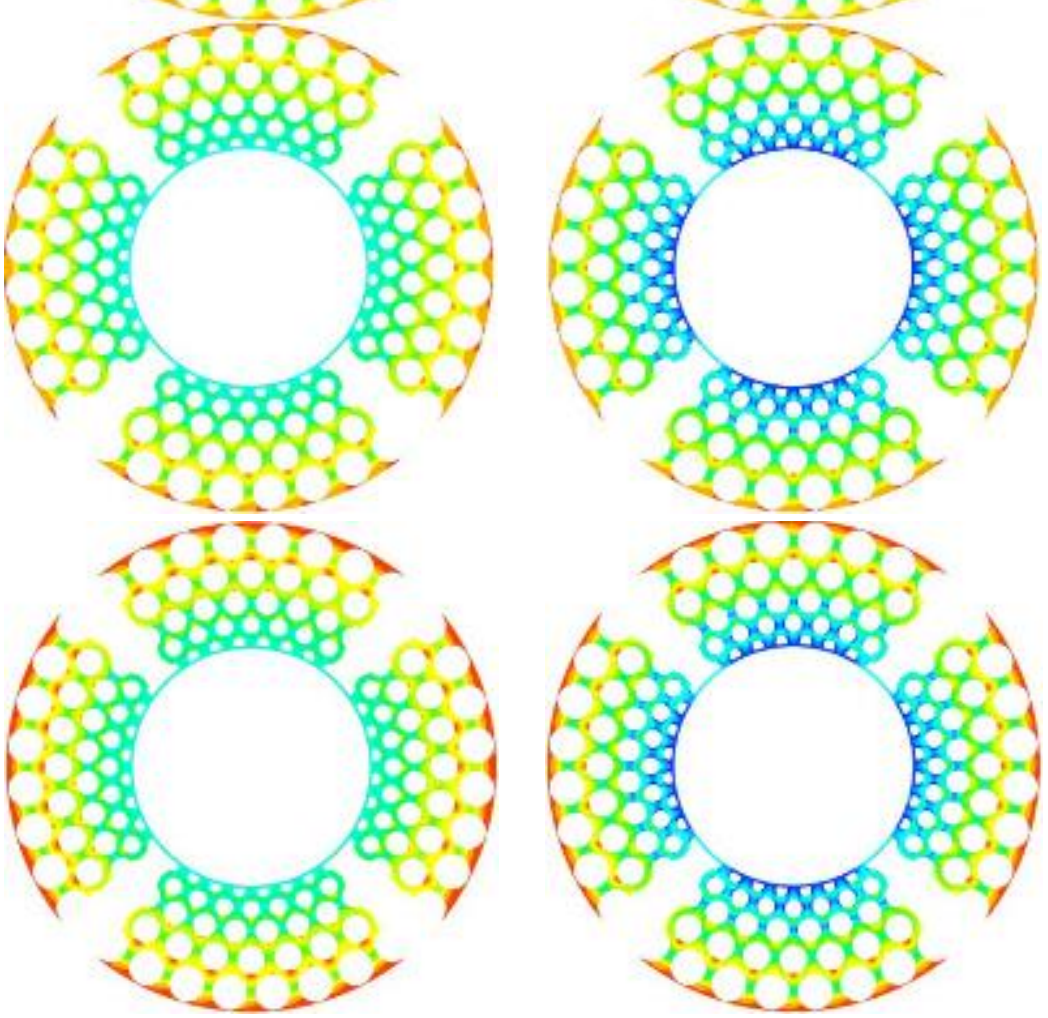

15
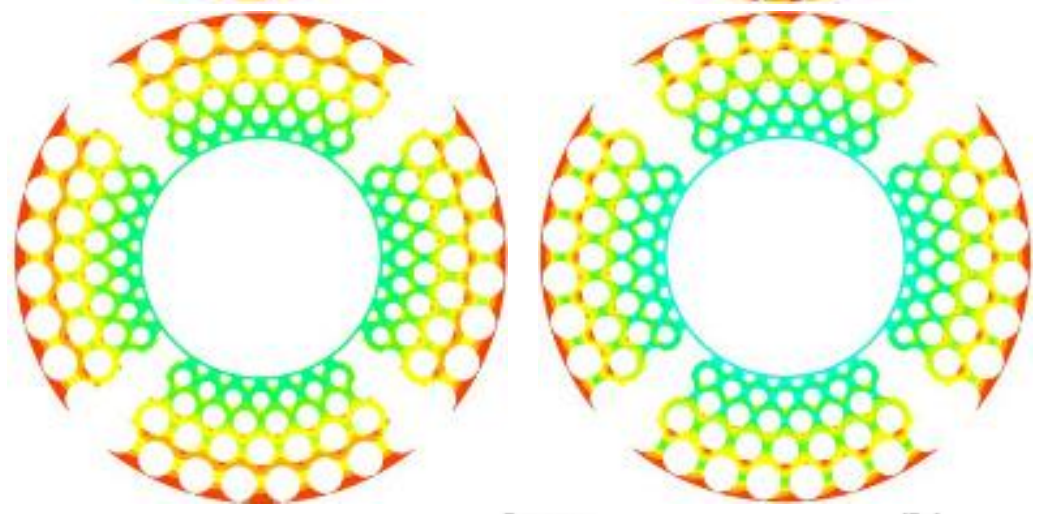

[Pa]

Pressure

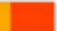

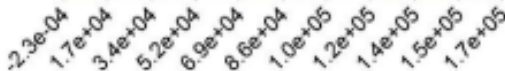

Figure 6: Pressure distribution for the top, middle and bottom discs at 5, 10 and $15 \%$ volume fractions at $100 \%$ VOP (average inlet velocity of $10 \mathrm{~m} / \mathrm{s}$ ). 
3.2.1 Effect of inlet volume fraction on local pressure characteristics at $100 \%$ valve opening position (VOP)

Figure 6 depicts the pressure field on the top, middle, and bottom discs of the trim at 5, 10 and 15\% volume fractions. Superimposed on the image for the top disc at $5 \%$ volume fraction are locations of planes A-F (also see Fig. 1 (e)) where numerical data of pressure, velocity and void fraction were extracted to obtain their profiles for further analysis. It can be seen in the figure (the top disc at $5 \%$ volume fraction in Figure 10) that pressure changes from $143 \mathrm{kPa}$ at the inlet (location A) to $63 \mathrm{kPa}$ at the outlet (location F) i.e. a pressure drop of $80 \mathrm{kPa}$. In comparison, the case of water only flow produced a decrease in pressure from $331.74 \mathrm{kPa}$ to $173.2 \mathrm{kPa}$, a pressure drop of $160 \mathrm{kPa}$ for the top disc. Moving axially downwards, it can be seen that progressively, there are increased pressure gradients in the middle and bottom discs. Radially, the staggered cylinder arrangement poses a flow resistance that results in a staged pressure reduction from inlet to outlet; the gradient in the axial direction is due to phase stratification. A similar trend in pressure gradient from the top to bottom is obtained for the 10 and $15 \%$ gas volume fraction cases. However, the outlet pressure at the bottom disk is essentially similar to the $5 \%$ volume fraction case. As a result, the inlet-outlet pressure drops are increased accordingly by 7 to $20 \mathrm{kPa}$ for the latter two cases relative to the same disk position (top, middle or bottom) at $5 \%$ inlet volume fraction. It can hence be concluded that as volume fraction of air increases, pressure drop across the trim increases. Figure 7 summarises these pressure variations for the three inlet gas volume fractions. Again, the gradients are primarily along the radial direction and the pressure field is axisymmetric as seen for single phase water flow.
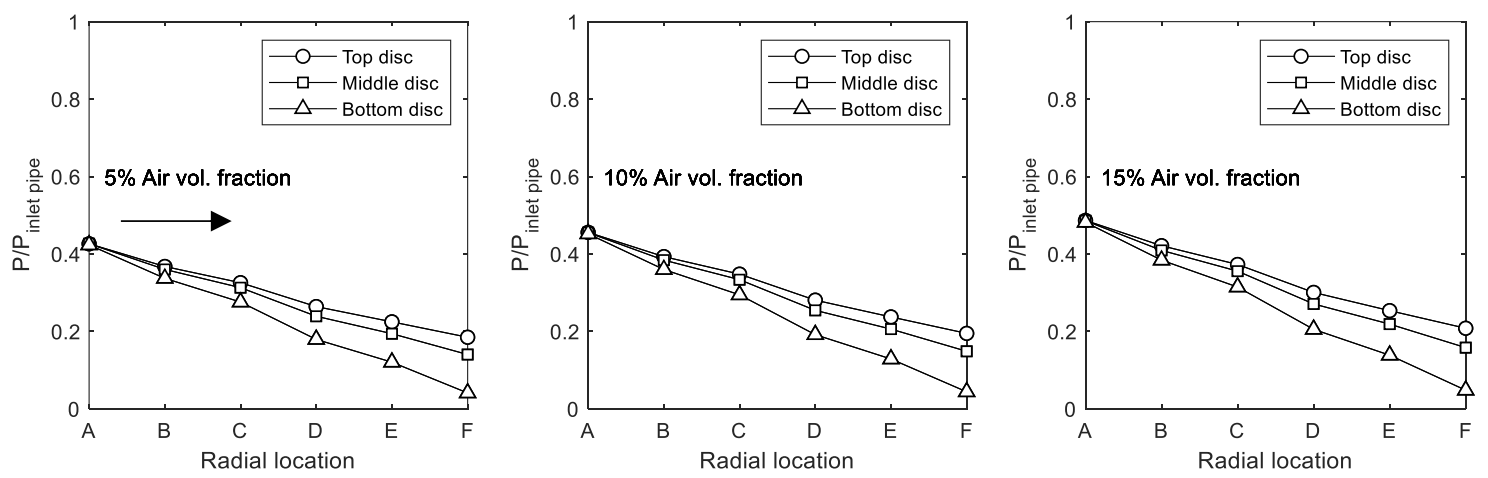

Figure 7: Mean radial pressure profiles for the top, middle and bottom discs at 5, 10 and $15 \%$ gas volume fractions at $100 \%$ VOP. Arrow indicates direction of flow in all plots.

Increasing volume fraction results in lower local pressure in the direction of flow i.e. from A to F. Initially, a rapid drop is noticed in the local pressure along the trim radius. Figure 8 (a) shows the radial pressure along the central flow path of a quadrant of the trim. It indicates a drop in pressure after each cylinder and recoveries just before encountering the next row of cylinders. Such a behaviour is due to the converging and expansion of the available flow areas. As compared to the $5 \%$ volume fraction, a $20 \%$ higher pressure drop is observed for the 10 and $15 \%$ volume fractions. In all, the singlephase water condition gives the lowest pressure drop across the trim when compared with the multiphase conditions. To quantify the effect of volume fraction on rate of pressure decrease in the direction of flow, the pressure drop values have been computed corresponding to each row. These were then non-dimensionalised and plotted as a function of row number as shown in Figure 8 (b). It is seen in the figure that the pressure drop trend is mixed. In some rows the pressure drops are higher for single phase flows whereas in some rows pressure drops are higher for the multiphase flow conditions. As the local velocities change in between the rows because of areas available for flow it has complex effects on the flow. The velocities depend on the areas available for the flow, resulting in different converging-diverging cross-sections depending on the row. Because of this, the pressure 
drop was plotted against the non-dimensional area ratio as shown in Figure 8 (c). For single-phase flow, pressure drop has an increasing trend against the area ratio [9], whereas under multiphase conditions, there is a minima of pressure drop noticed at the area ratio of 1.13 and on either side of this value the pressure drop seems to increase. Furthermore, the figures show that there is an increase in pressure drop in row 5 . The pressure drop is directly related to the area available for flow in the different rows. In row 5 , the total surface area around the flow path is higher than row 4 . Therefore, there is a higher resistance to flow at row 5.

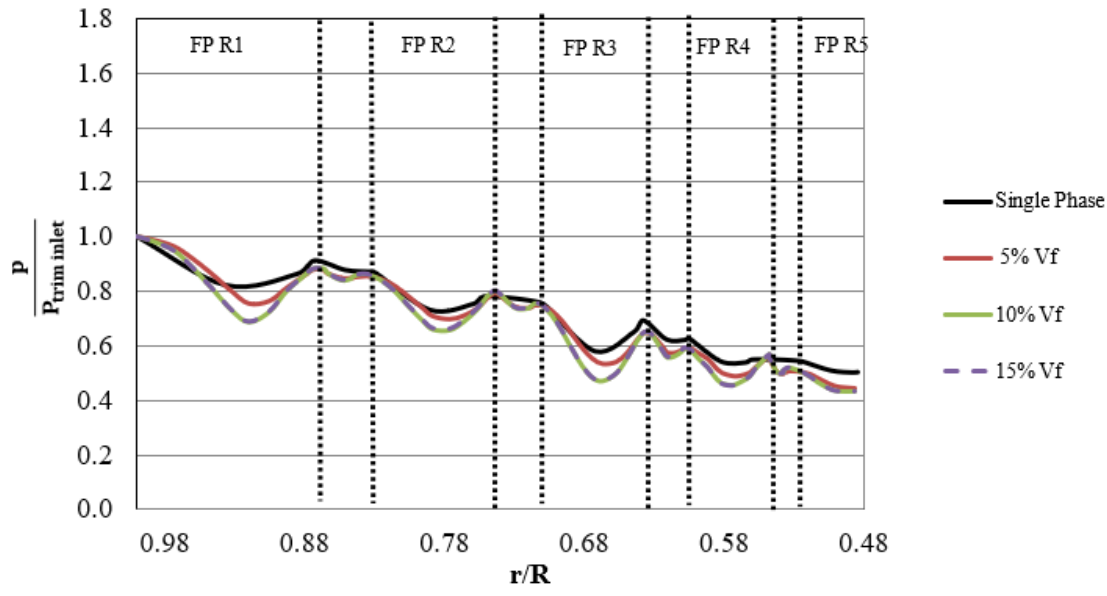

(a)

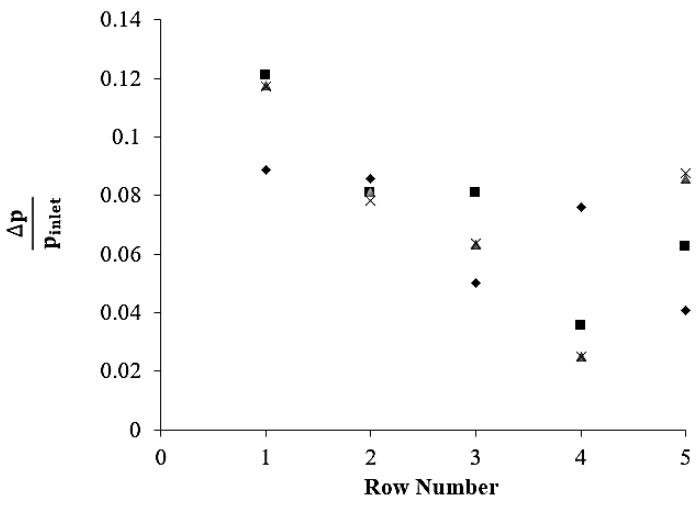

(b)

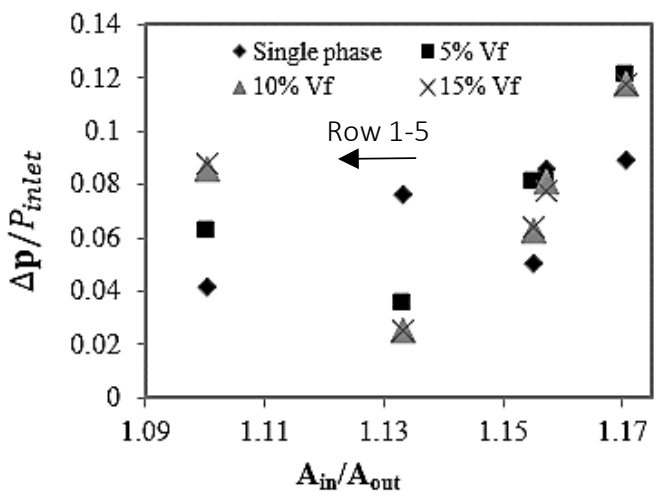

(c)

Figure 8: (a) Pressure distribution at different air volume fractions through different rows for the central flow path at $100 \%$ VOP. (b) Pressure drop values at different air-water mixture flow volume fractions through different rows at $100 \%$ VOP (c) Pressure drop values at different air-water mixture flow volume fractions against area ratios.

\subsubsection{Local water velocity field as a function of inlet volume fraction at $100 \%$ valve opening position}

The corresponding water velocity fields in the gas-liquid multiphase flow cases for the top, middle and bottom discs have been plotted and are shown in Figure A1. The local water velocities have increased with increasing volume fraction and the velocities appear to be non-uniformly distributed at the $10 \%$ and $15 \%$ inlet volume fraction conditions, especially at the top disc location. In comparison, single-phase water flow exhibits more uniformly distributed velocity profiles (see Figure 4). The nonuniformity present at the multiphase conditions is due to local velocity differences (slip) between the two phases. The multiphase interaction causes greater pressure losses within the trim due to interfacial friction that results from phase slip. This slip does not occur in single-phase hence lesser pressure gradient as well as more uniform distribution from inlet to outlet of the trim. To quantify the non-uniformity in the multiphase cases, non-dimensional velocity profiles were plotted for each quarter as shown in Figure 9. To obtain a baseline for comparison, the inlet velocity of $10 \mathrm{~m} / \mathrm{s}$ was 
used for non-dimensionalisation. The non-dimensional profiles show that on getting to the middle and bottom of the valve, the velocities become more axisymmetrically distributed with respect to the quadrants. The velocities for each gas volume fraction, quadrant and disc peaks at radial location $D$. For the top disc, a progressive decrease occurs afterwards until the exit of the trim at location $\mathrm{F}$. Conversely, for the middle and bottom discs, the exit velocity at location $\mathrm{F}$ increased rather than decrease as was experienced for the top disc at all three inlet gas volume fractions. Quantitatively, for the top disc at $5 \%$ volume fraction, the local water velocity values varied from $1.81 \mathrm{~m} / \mathrm{s}$ at location $A$ to $3.83 \mathrm{~m} / \mathrm{s}$ at location $F$ with a peak of $4.61 \mathrm{~m} / \mathrm{s}$ at location $D$. These are shown in Figure 9 and again, it is worth mentioning that at $5 \%$ volume fraction, the profiles were mostly axisymmetric from top to bottom. However, the water velocities at the exit increased to 4.69 and $6.05 \mathrm{~m} / \mathrm{s}$ for the middle and bottom of the discs.
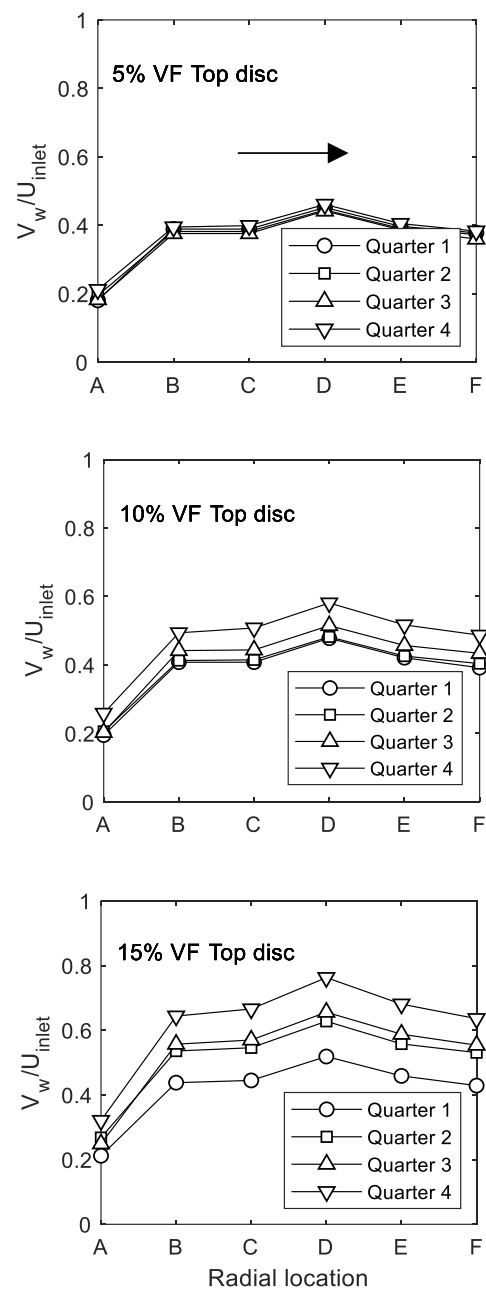

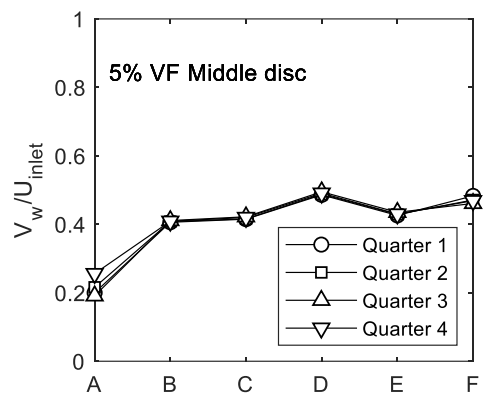

(a)

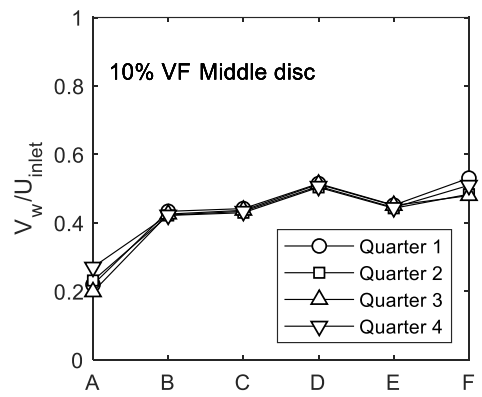

(b)

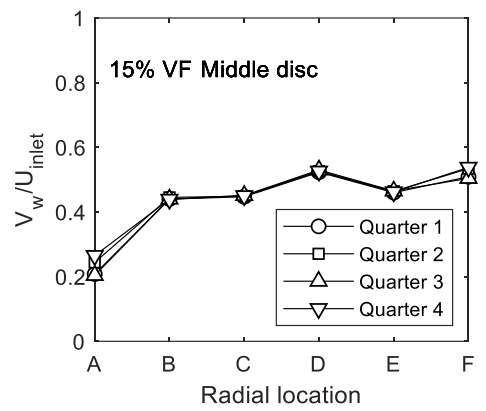

(c)
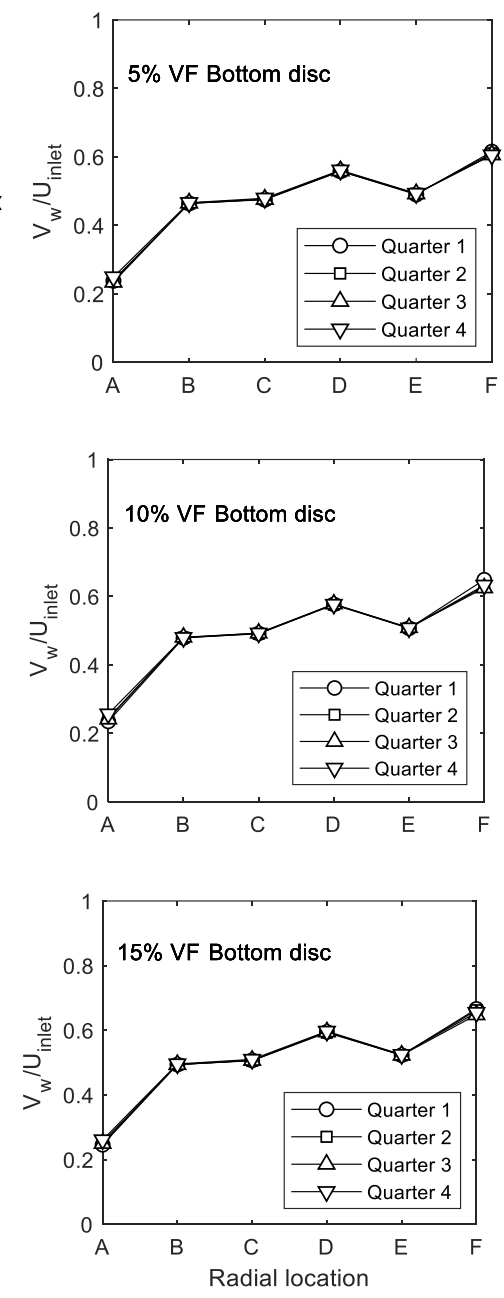

Figure 9: Quarterly radial water velocity profiles for the top, middle and bottom discs, at (a) 5, (b) 10, and (c) $15 \%$ air inlet volume fractions respectively and at $100 \%$ valve opening (mean inlet velocity of $10 \mathrm{~m} / \mathrm{s}$ ). Arrow indicates direction of flow in all plots.

A similar water velocity trend occurs at $10 \%$ volume fraction from top to bottom. For the $15 \%$ volume fraction condition, largest velocities occurred at the $4^{\text {th }}$ quadrant which ranged from $3.2 \mathrm{~m} / \mathrm{s}$ at the inlet at location $A$ to $6.36 \mathrm{~m} / \mathrm{s}$ at the exit at location $F$ with a peak of $7.63 \mathrm{~m} / \mathrm{s}$ at location D. These values were a third lower radially throughout the trim for the $1^{\text {st }}$ quadrant. The $1^{\text {st }}$ to $4^{\text {th }}$ quadrants showed similar velocities in each quadrant and ranged from $2.04 \mathrm{~m} / \mathrm{s}$ to $5.38 \mathrm{~m} / \mathrm{s}$ at the exit with a marginally lower velocity of $5.29 \mathrm{~m} / \mathrm{s}$ at location D. Progressive increase in local water velocities occurred for the bottom disc at the $15 \%$ volume fraction condition, and these ranged from $0.261 \mathrm{~m} / \mathrm{s}$ 
to $0.655 \mathrm{~m} / \mathrm{s}$ at the exit. For all cases, the velocity profiles at the middle and bottom discs are symmetric and the effect of inlet gas volume fraction is limited to the top few discs. To summarise, it was generally observed that in comparison with single phase, water velocity values under multiphase conditions are slightly lower. Also, losses can be incurred at the gas-liquid interface due to the occurrence of slip between the phases, as the gas travels at a faster rate than the liquid. The air velocity fields at the three disc locations are depicted in Figure 10 and indicate that the air velocities are almost very close to the water velocities. The velocity values vary from $3.85 \mathrm{~m} / \mathrm{s}$ to $7.24 \mathrm{~m} / \mathrm{s}$. it has been seen that because of complex geometric effects at some places air velocity is higher than the water velocity (point $\mathrm{A}$ and $\mathrm{E}$ ) and at some places lower than the water velocity. Further analysis of the non-uniformity in phase-slip is discussed in the next section.

Inlet vol.

fraction

[\%]

\section{Top disc}

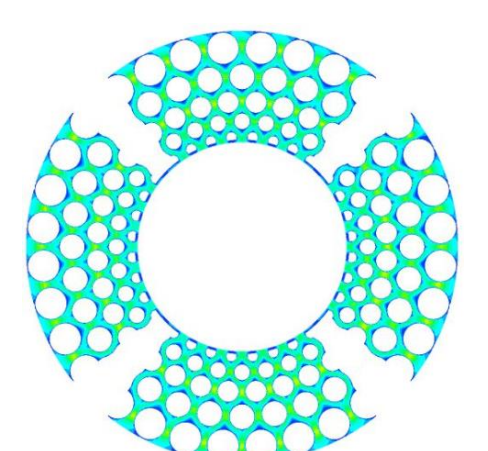

5

10

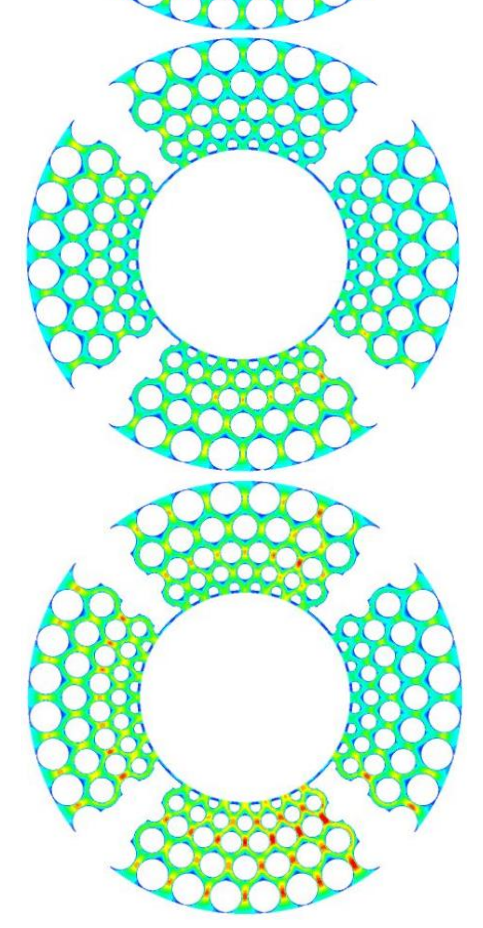

15
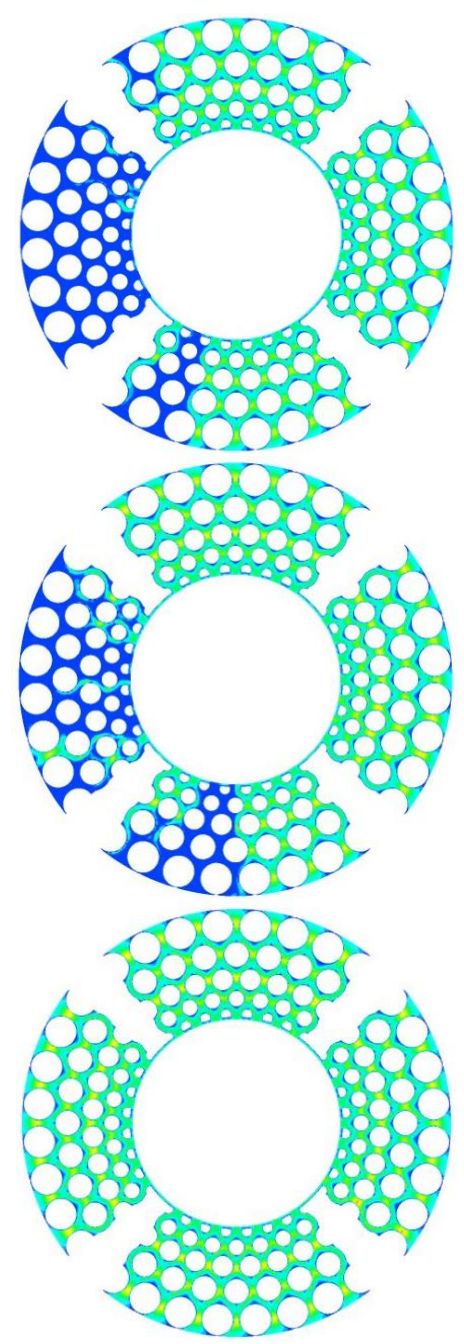

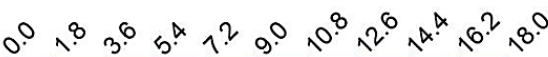

Bottom disc
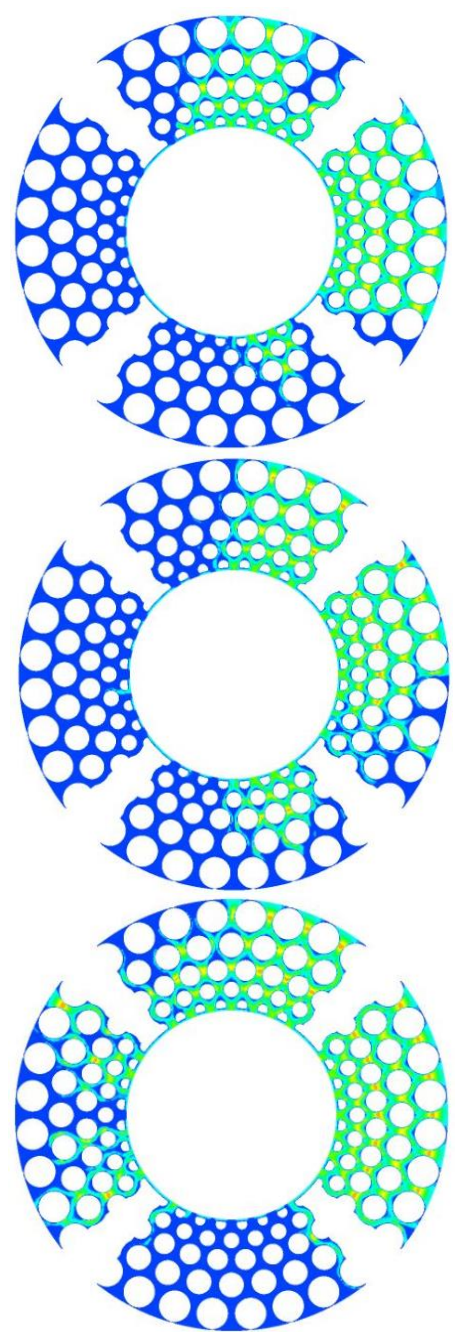

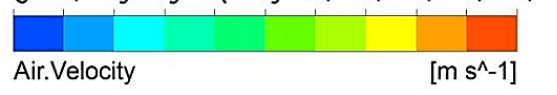

Figure 10: Air velocity profiles at the top, middle and bottom discs, at 5, 10, and $15 \%$ volume fractions respectively and at $100 \%$ valve opening (average inlet velocity of $10 \mathrm{~m} / \mathrm{s}$ ).

\subsubsection{Inlet volume fraction effect on local air velocity field at $100 \%$ valve opening position}

The air velocity values have also shown increasing trend with increase in volume fraction, however at some locations (i.e. the left and bottom left locations of the middle and bottom discs) a reduction in 
velocity is also seen. These are shown in Figure 10. This indicates that a complex geometry affects the gas velocity field in quite a complex manner, and this results in a re-arrangement of the velocity field with increasing volume fraction. One important aspect that can be seen here is that the ratio of water velocity and air velocity is not constant, and the variation is quite complex. The local air volume fraction distribution is also expected to significantly depend on the slip ratio and overall inlet volume fraction. It would be important to explore slip variation and spatial distribution of void fraction within the geometric complexity of the trim. A drift-flux type distribution coefficient was derived to quantify the phase non-uniformities and it is presented subsequently in Section 3.4.2. To quantitatively establish the effect of velocity differences between the air and water against the pressure field characteristics, the ratio of water velocity to the air velocity has been calculated and plotted as shown in Figure 11. The water velocity is mostly higher than the air velocities in between the rows. As the flow passes between the cylinders in each row, $V_{w} / V_{a}$ starts increasing until it reaches the exit of the row. In the region between different rows, $V_{w} / V_{a}$ decreases and is nearer to unity at the entry of the rows. The pressure profile however experiences progressive decrease along the trim, while that of the velocity ratio experiences a gradual increase, with $V_{w} / V_{a}$ peaks occurring on reaching the cylinder rows. Thus, the complexity of geometry is seen to significantly affect the flow velocities corresponding to different phases resulting in areas of high pressure and low velocity when flow passes through the cylinder rows. However, in between the rows, there are areas of low pressure and high velocity occurrences and slip ratio can also be less than unity. As a result, completely different flow regime can be obtained. These are considerations that need to be considered during the design process of multiphase control valves.

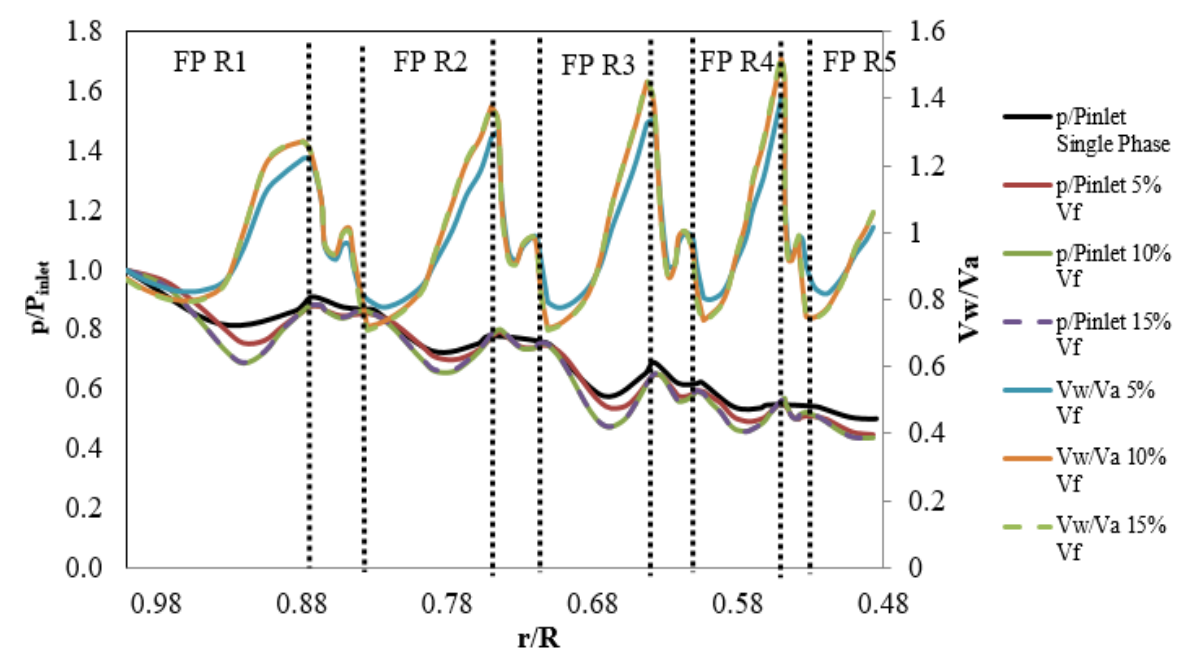

Figure 11: Variation of top disc slip and pressure drop values at $100 \%$ valve opening at different air-water mixture flow volume fractions against radius ratio.

\subsubsection{Local air volume fraction distribution at $100 \%$ valve opening position}

The air volume fraction distribution corresponding to $100 \%$ valve opening is shown in Figure 12 which reveals that the volume fraction distribution is not axisymmetric, indeed it is non-uniform in all the quadrants. The local void fraction is seen to be as high as $40 \%$ in some quadrants at the top and middle discs for the $5 \%$ air inlet volume fractions. 

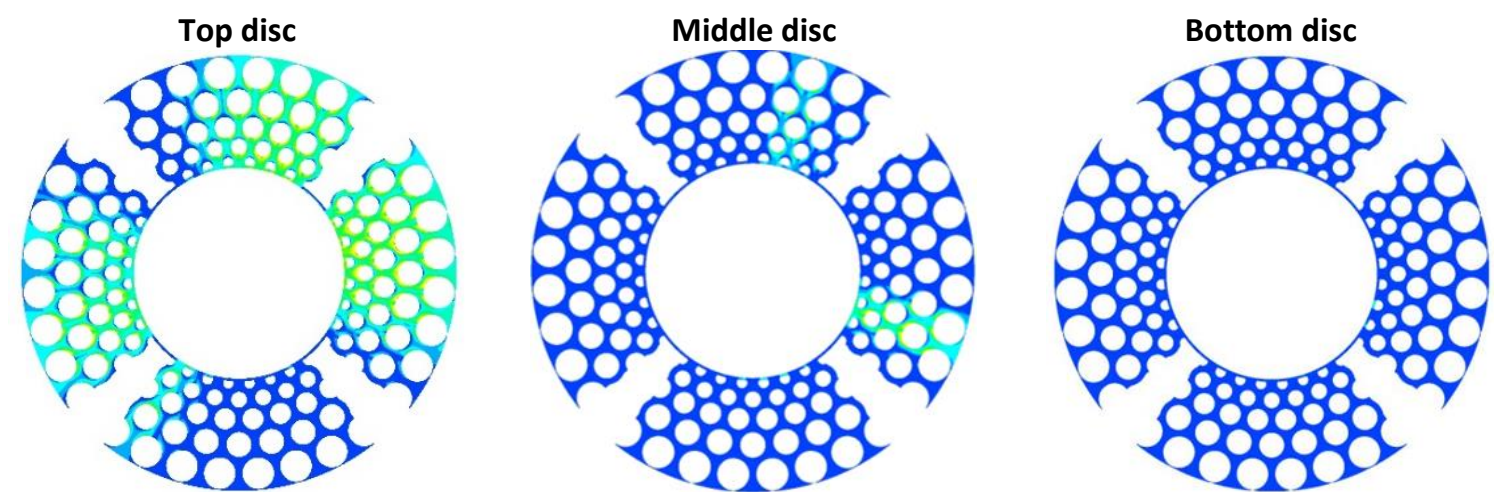

Axial view

5

10

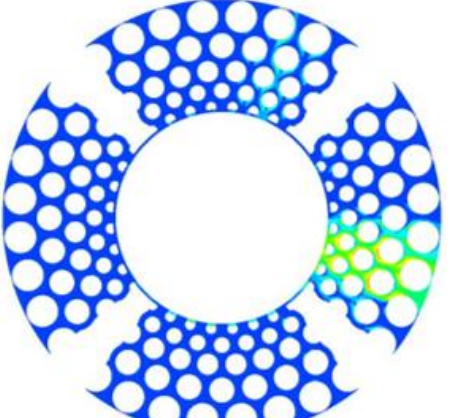

政

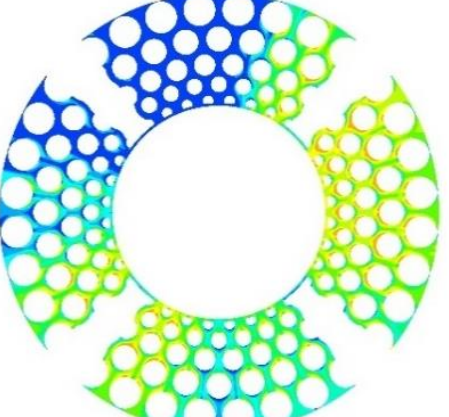

10
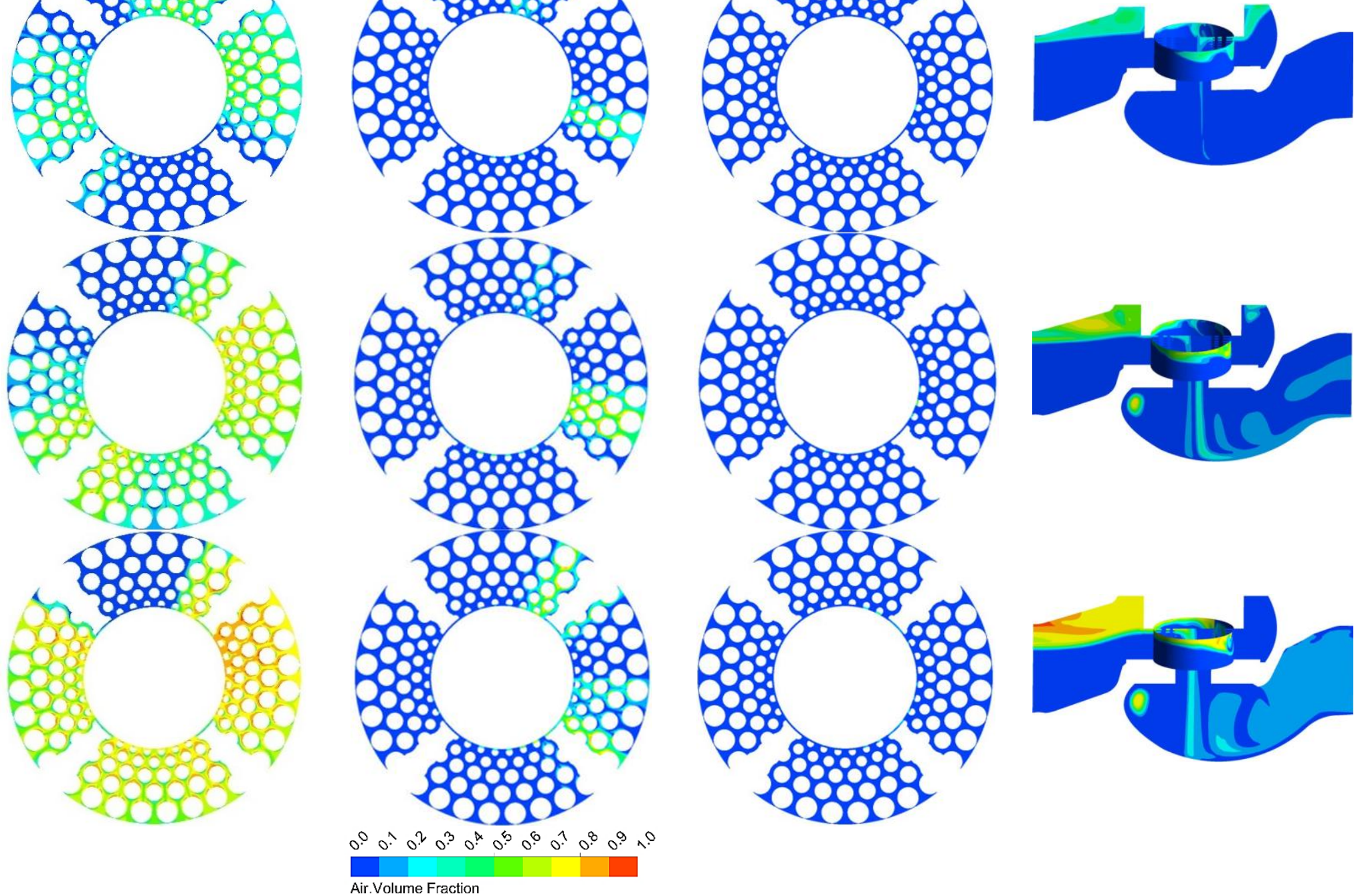

15

Air.Volume Fraction

Figure 12: Volume fraction distribution for air-water mixture flow at 5,10 , and $15 \%$ inlet air volume fraction for the bottom, middle and top disc locations at $100 \%$ VOP. 
1 A scrutiny of volume fraction contours indicates that at $10 \%$ overall volume fraction, the air volume

2 fraction across the disc varies considerably and is non-axisymmetric. In all the quadrants the 3 distributions are different. Higher local void fractions are observed in more quadrants with increasing 4 inlet gas volume fraction to $10 \%$ and $15 \%$ and there are areas in the disc which are predominantly 5 occupied by the gas phase at the top and middle discs. At highest volume fraction of $15 \%$ there is more 6 homogeneity in the volume fraction distribution with the gas phase being dominant at the top disc 7 than at lower volume fraction values. In all three inlet gas fraction cases, the bottom disc is almost 8 entirely occupied by the liquid phase (as shown by the axial view images on the right of Figure 12). 9 This is called phase stratification. However, as shown in Figure 10, the gas velocity can be high at the 10 bottom location despite its low volume fraction. To capture the effect of both local velocity and phase 11 fraction, the concept of local volumetric concentration is usually used, and it is represented by a "distribution coefficient". This will be discussed in more detail in section 3.4.2. In comparison to singlephase liquid flowing in the system, the above analyses clearly indicate that the flow pattern changes considerably when multiphase flow is concerned, resulting in severe velocity-related effects as well as volume fraction-related effects.

\subsection{Effect of volume fraction on local pressure at $60 \%$ valve opening position}

The characteristics of flow and valve performance in partial opening conditions can be different impacted. It is noteworthy that the same disc in partial opening conditions can have entirely different flow behaviour as compared to $100 \%$ open condition, and may result in completely changed pressure, velocity and volume fraction profiles. Current design methods do not take this difference into consideration and assume that the flow conditions corresponding to the discs do not change. This work provides a benchmark for partial valve opening positions to be included. To quantify the differences in the local distribution of phase velocities, pressure and gas volume fraction, an investigation done with a partial valve opening position of $60 \%$ is presented in the following sections.

\subsubsection{Inlet air volume fraction effect on local pressure distribution at $60 \%$ valve opening position}

Under partial valve opening conditions, especially for multiphase flows, the flow condition may register significant variation owing to complex trim geometry. Keeping this in view, the local flow field characteristics (pressure, water velocity, air velocity, and local volume fraction distribution) have been evaluated at $60 \%$ valve opening condition. As a result, not all the three discs previously shown in the $100 \%$ VOP are exposed to the flow - only the middle and bottom discs. Hence, these are the two available for visualisation and analysis. Figure A2 (a) shows the pressure distribution at the middle and bottom discs for the 5, 10, and $15 \%$ inlet volume fraction conditions at $60 \%$ VOP. It shows that for the three inlet volume fractions, the pressure distribution is axisymmetric with respect to the disc quadrants. At $5 \%$ air volume fraction, the local pressure at the inlet i.e. location A was $227.4 \mathrm{kPa}$ which reduced to $63 \mathrm{kPa}$ at location $\mathrm{F}$ at the outlet. In comparison to the $100 \%$ valve opening, the pressure reduction from inlet to outlet at the same condition and disc location was $146 \mathrm{kPa}$ to $47 \mathrm{kPa}$ respectively. Therefore, a larger pressure loss (of $164.4 \mathrm{kPa}$ ) occurs at $60 \%$ VOP than that for a fully open valve $(99 \mathrm{kPa})$. The trend is replicated at the 10 , and $15 \%$ air inlet volume conditions and it can be inferred that partially open valve conditions result in higher pressure losses. This comparison is evident when one looks at the dimensionless pressure $P / P_{\text {inlet }}$ profiles given in Figure 11 and Figure 13 (a) at $100 \%$ and $60 \%$ VOPs respectively. While for $100 \%$ VOP, $P / P_{\text {inlet }}$ falls from 1 to around 0.4 , this value ranges from 1 to around 0.25 for the $60 \%$ VOP signifying a larger pressure drop in the latter VOP for single-phase water and all inlet volume fractions for the multiphase conditions. 
For further quantifying the effect of volume fraction on the rate of pressure reduction radially across the trim, dimensionless pressure drop values were calculated for each row. This was then nondimensionalised and plotted for each row as shown in Figure 13 (b). It is seen in the figure that the pressure drop exhibits a mixed trend. While in some rows the pressure drops are higher for single phase flows in others, the pressure drops are higher for the gas-liquid flow conditions. The local velocities change between rows. This is because there are different areas available for flow and this results in rather complex effects on the flow behaviour. To evaluate these effects, the pressure drop was plotted against the area ratios, and are as shown in Figure 13 (c). For single-phase flow, pressure drop has an increasing trend against the air ratio whereas under multiphase the lowest pressure drop is noticed at the area ratio of 1.13 and on either side of this value the pressure drop seems to increase.

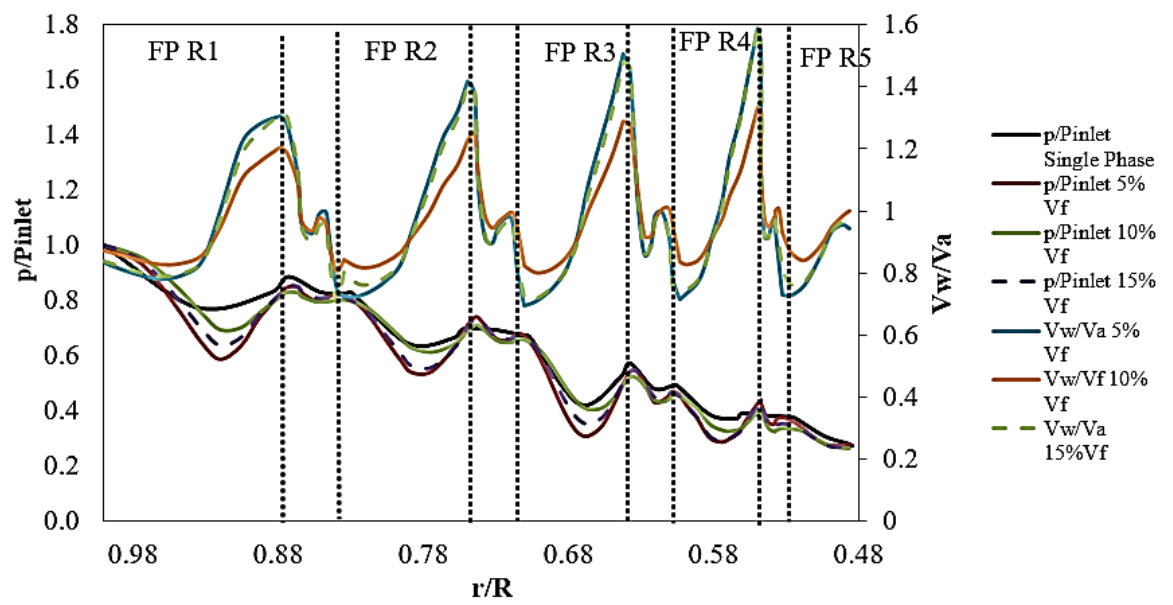

(a)

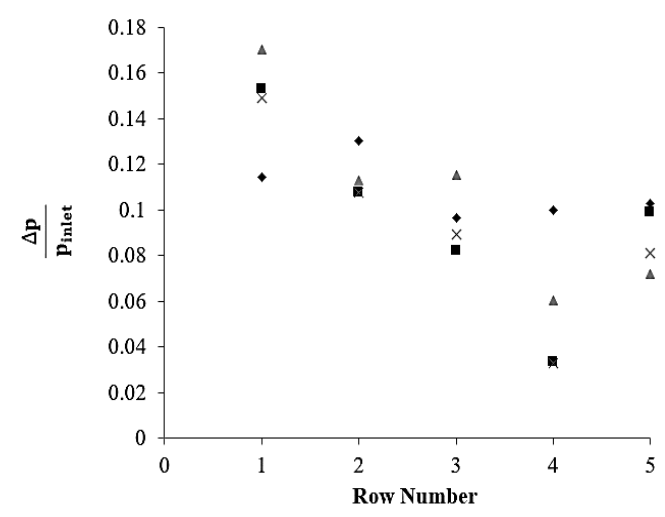

(b)

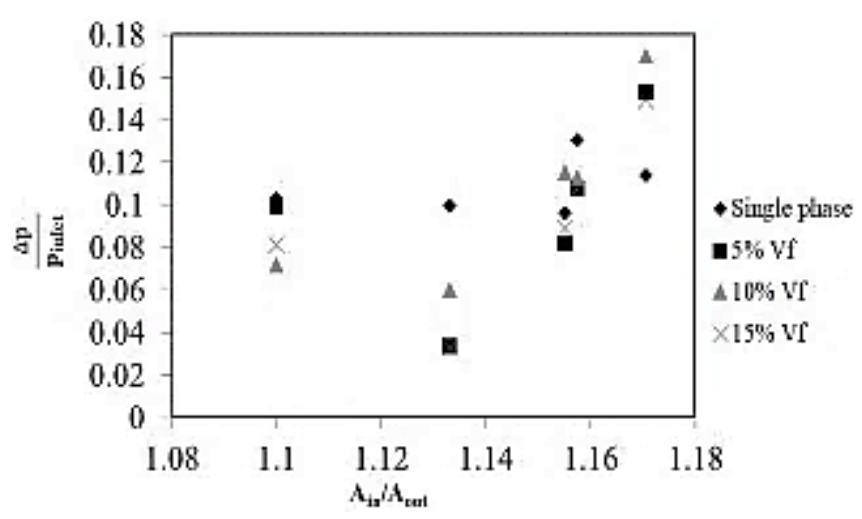

(c)

Figure 13: (a) Variation of top disc slip and pressure drop values at 60\% VOP for different air-water mixture flow volume fractions against radius ratio for the middle disc's central flow path. Dimensionless pressure drop at different air volume fractions (b) through different rows (c) at different inlet/outlet area ratios at $60 \%$ VOP (legend entries are the same for figures $b$ and $c$ ).

\subsubsection{Characteristics of Local water and air velocity fields as a function of inlet volume fraction at} $60 \%$ VOP

The water velocity fields at the middle and bottom discs corresponding to $60 \%$ VOP are shown in Figure $A 3$ in the Appendix. These show that the water velocity distribution across the middle and bottom of the trim are axisymmetric, similar at both axial locations and show a slight increase with increase in void fraction especially at $15 \%$ inlet air volume fraction where the increase is more apparent. In comparison with the $100 \%$ VOP for the same inlet flow rates, maximum velocities of up to $24 \mathrm{~m} / \mathrm{s}$ are obtained at the middle disc of the $60 \%$ VOP case while only around $13 \mathrm{~m} / \mathrm{s}$ maximum occurs in the top disc of the $100 \%$ VOP case (see Figure A1). Essentially, valve opening has a significant 
effect on not only the magnitude of water velocities in the trim, it also affects the spatial distribution with lower VOP producing more uniform water velocity distribution within the trim disc especially at the upper locations within the trim. The left half Figure 23 shows the distribution of air velocities within the middle and bottom discs of the trim at 60\% VOP. The lowest velocities are at Quarter 4 (the lower part of each disc) and towards the bottom of the trim. This means that the air is displaced by the water at the entrance, forced towards the opposite end of the trim, and leave the trim radially inwards at the top discs leading to a rather stratified gas-liquid regime in the trim. The distributions show that as air volume fraction increases, the gas-liquid stratification increases as evidenced by the increasing distribution of the largest air velocities at the middle disc compared to the lowest at the bottom trim discs. Furthermore, the stratification means that the circumferential averaged volume fractions at $60 \%$ opening at the top will decrease as all air will have to flow through a smaller area and more mixing will occur.

Inlet air volume fraction

[\%] 5

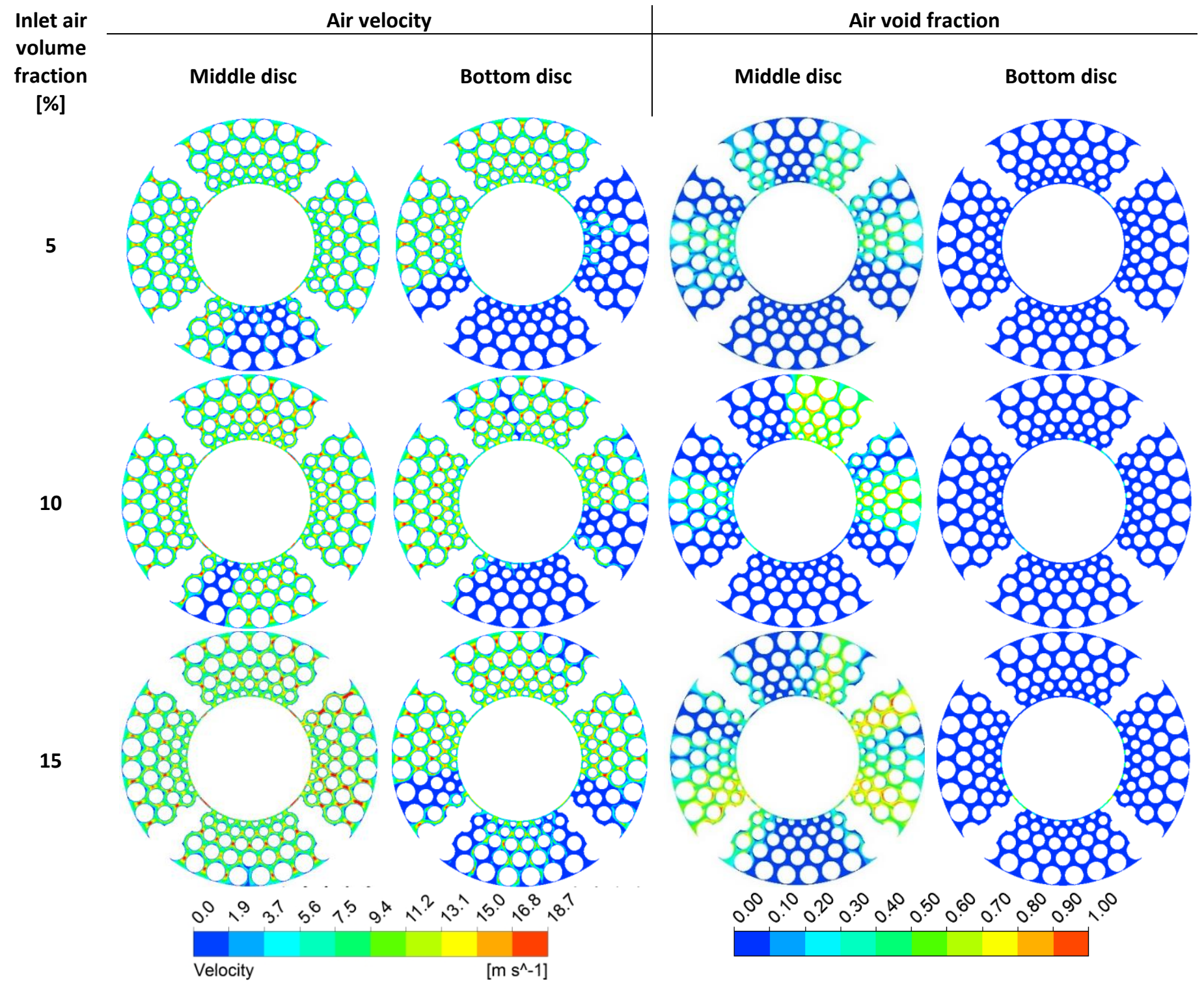

\begin{tabular}{l|l|} 
& \\
Bottom disc
\end{tabular}

Air void fraction

Figure 14: Air velocity and void fraction distributions at the middle and bottom discs, at 5, 10, and 15\% volume fractions respectively and at $60 \%$ valve opening (average inlet velocity of $10 \mathrm{~m} / \mathrm{s}$ ).

As seen in the earlier section, air appears to flow in a stratified manner along with the water phase.

To visualise how it is spatially distributed, contours of the air volume fraction at the middle and bottom 
discs were obtained as shown on right half of Figure 14. At the middle disc for all inlet volume fraction cases (i.e. 5, 10, and 15\%), it can be seen that at the entrance region (Quadrant 4, lower part of each figure) the volume fraction is between 0 and 0.1 with the air being made to flow at the sides and opposite end of the entrance zone. In these parts, the air fraction increases with inlet volume fraction with local values rising from 0.5 to 0.8 at $15 \%$ inlet volume fraction. At the bottom disc, the local air volume fraction for all three cases is less than 0.05 indicating stratification of the phases with a dominant liquid phase towards the bottom. To compare differences in local gas volume fraction distribution as a result of valve opening position, plots have been produced for the volume fraction for the central flow path for a quadrant of the top disc. These are shown in Figure 15. It indicates there is a general increase in volume fraction with a decrease in valve opening position, with the $60 \%$ VOP exhibiting higher values (e.g. $0.4-0.8$ for $5 \%$ VF) than the corresponding values $(0.25-0.6$ for $5 \% \mathrm{VF})$ at $100 \%$ VOP. Additionally, there is increase in air volume fraction as the flow proceeds from inlet to the outlet of the trim. This is because of expansion going from inlet to outlet due to pressure drop (plots shown in Figure 11 and Figure 13 (a) for $100 \%$ and $60 \%$ respectively). There is a higher pressure drop for the $60 \%$ VOP in comparison to the $100 \%$ VOP case, resulting in the increased local volume fraction along the flow path for $60 \%$ VOP.

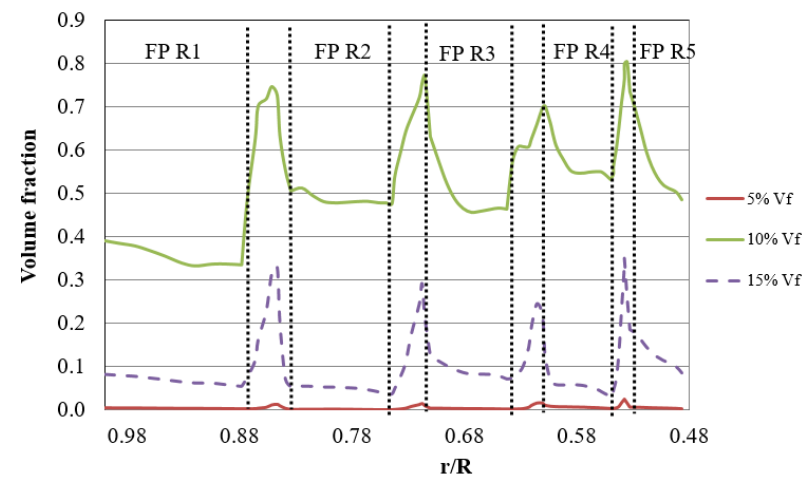

(a)

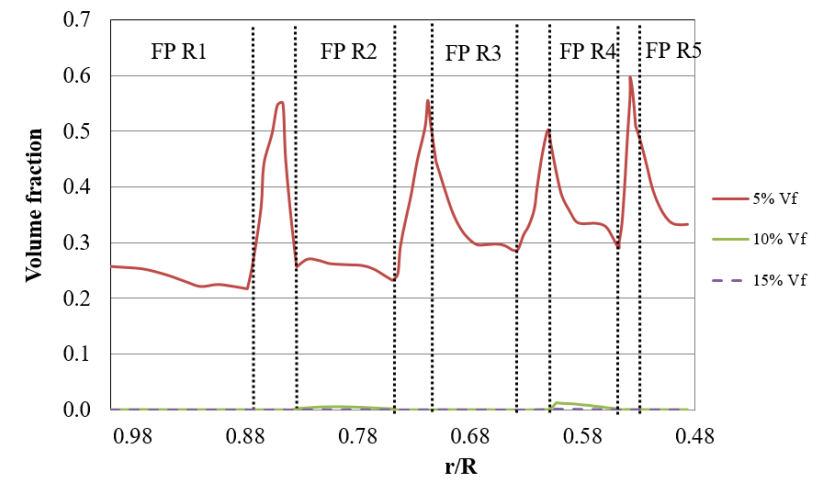

(b)

Figure 15: Comparison of local volume fraction profile for a quadrant of the top disc's central flow path (a) $60 \%$ VOP (b) $100 \%$ VOP

\subsection{Prediction of local flow parameters incorporating the inlet volume fraction}

\subsubsection{Local air volume fraction and flow coefficient}

Local air volume fraction at the top, middle and bottom discs were extracted from the CFD simulations at planes A-F (see Figure 1 (d) for locations of the planes). The value of each quantity that corresponds to row 1 for example are those entering the row and extracted from plane $A$, those entering row 2 are those extracted from plane $B$, and so on. As a result, the air volume fraction data is available at 5 rows of every quarter of three discs within the trim. These air volume fractions are denoted with the

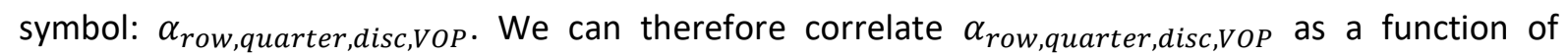
geometry (i.e. location within trim) and inlet gas volume fraction by assuming the following power law relationship:

$$
\alpha_{\text {row }, q u a r t e r, \text { disc }, \text { VOP }}=\frac{W(\text { Row }+1)^{q}(\text { Quarter }+1)^{r}}{(\text { VOP }+1)^{s}(\text { Disk }+1)^{t}} \alpha_{\text {inlet }}^{u}
$$

where Row, Quarter and Disk are positive integers describing their respective locations within the trim. The numbering for each of these is given in Figure 1 (c) and (d). VOP is the valve opening position and takes values between 0 and 1 (i.e. between 0 and 100\%). Therefore, for the current work, the two 


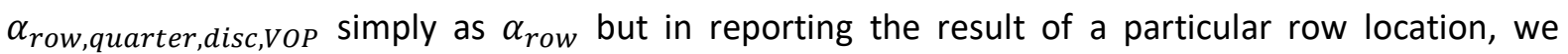
recommend writing out the subscripts in full to avoid ambiguity. The coefficient $W$ and indices are $q$, $r, s, t$, and $u$ are regression constants determined by fitting the CFD data to the right-hand side of the equation using the least squares method. The application of power law correlation methods with least squares regression is a common approach to related independent variables with output parameters and there are numerous examples of this approach in gas-liquid two-phase flow research [33], [34]. Applying the method yielded the following relationship where information of the inlet volume fraction is an input in determining the volume fraction distribution within the different rows and discs in the trim:

$$
\alpha_{\text {row }}=\frac{93.37(\text { Row }+1)^{0.16}(\text { Quarter }+1)^{1.01}}{(\text { VOP }+1)^{5.00}(\text { Disc }+1)^{1.78}} \alpha_{\text {inlet }}^{1.02}
$$

10

11

For single-phase flow where only water flows through the trim, $\alpha_{\text {inlet }}=0$, thereby making $\alpha_{\text {row }}=0$. To illustrate the use of Equation (7), suppose we would like to determine the area averaged void fraction for the $100 \%$ VOP case with $5 \%$ inlet volume fraction at row 3 of quarter 4 of disc 1 (i.e. the top disc), the input variables will be Row $=3$, Quarter $=4$, Disc $=1, \operatorname{VOP}=1$ and $\alpha_{\text {inlet }}=0.05$. Substituting these into Equation (7) yields $\alpha_{\text {row } 1, \text { quarter } 4 \text {,disc } 1, V O P 1}$ or $\alpha_{1,4,1,1}=0.2565$ for $5 \%$ inlet volume fraction. This value deviates from the CFD measured void fraction for that row location $(0.2685)$ by $4.47 \%$, which is acceptable. Figure 16 (a) shows that the larger local volume fractions (obtained at the top discs) are well predicted by this equation and within $30 \%$ of the CFD values. However, significant deviations are obtained at some lower volume fractions, which as will be shown in the next section, does not greatly impact the prediction of $K_{V_{\text {row }}}$. Nevertheless, as indicated by the correlation coefficient $\left(R^{2}\right)$ value of 0.8121 , the equation well agrees with the general trend of the entire dataset as shown in Figure 16 (a).
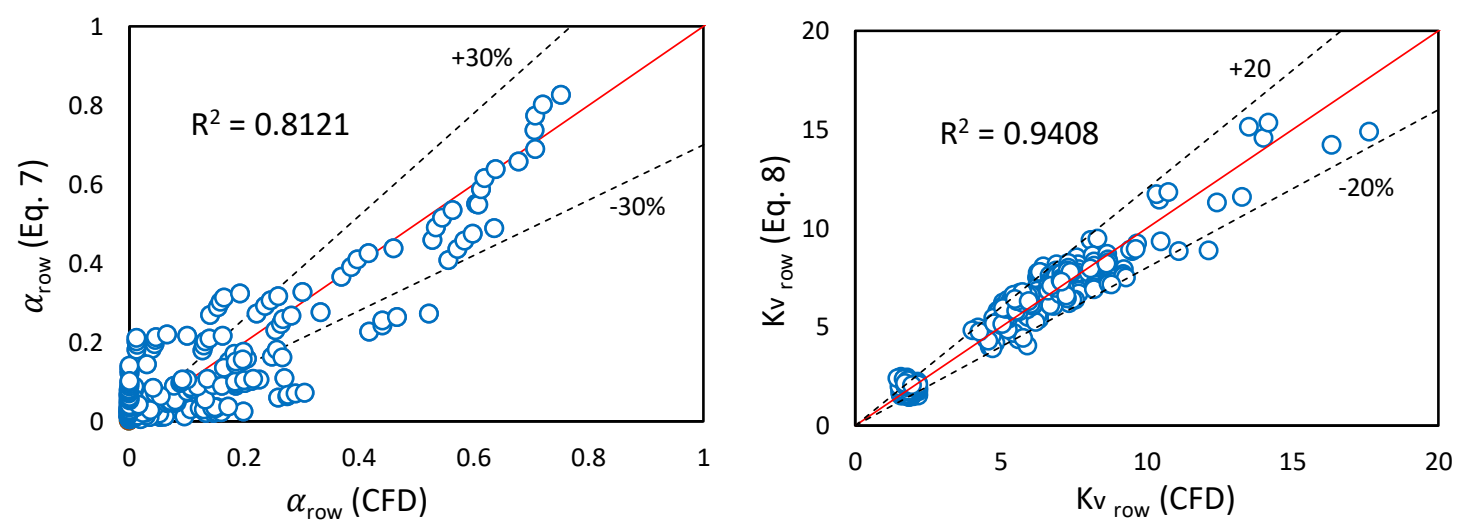

Figure 16: (a) Comparison between $\alpha_{\text {row }}$ obtained by CFD and Equation (7) (b) Comparison between local flow coefficient for each row obtained by CFD and calculated using Equation (8)

To calculate the local flow coefficient for each row of the trim i.e. $K_{V_{r o w}}$ area weighted averages for the local pressures as well as mixture density and volumetric flow rate of the mixture were extracted from the CFD data for planes $\mathrm{A}-\mathrm{F}$ (see Figure 1 (e) for details of the planes). The pressure drop $\triangle \mathrm{P}$ for row 1 for example was calculated as the difference in pressure from plane $A$ and $B$, that of row 2 is the difference in pressure of plane $B$ and $C$ and so on. On the other hand, the mixture densities and volumetric flow rates used were those entering row 1 at plane $A$, row 2 values are those at plane $B$, and so on. As a result, these give characteristic pressure drop, mixture density and volumetric flow rate in that row. From these, the local flow capacity $K_{v}$ for each row was then calculated using Equation (2). In order to correlate the local flow coefficient with disc/row/quarter location, we 
propose a relationship that includes the effect of the local gas volume fraction of the row $\alpha_{\text {row }}$ (given by Equation (7)). This is done by incorporating into Equation (1), local geometrical information (i.e. which quarter, disc, or row or what VOP) and the volume fraction at the row of interest. Fitting the $K_{V_{\text {row }}}$ data to the relationship, the following equation was achieved:

$$
K_{V_{\text {row }}}=7.50 Q_{\text {mix }} \sqrt{\frac{\text { Disk }+1}{\Delta \mathrm{P}}} \frac{(\text { VOP }+1)^{6.48}\left(\alpha_{\text {row }}+1\right)^{2.97}}{(\text { Row }+1)^{0.14}(\text { Quarter }+1)^{1.23}}
$$

Figure 16 (b) shows a comparison between $K_{V_{\text {row }}}$ predicted by this equation and the CFD values. As can be seen, a vast majority of the predicted values fall within a $\pm 20 \%$ margin of the CFD values including producing a strong correlation $\left(R^{2}\right.$ value of 0.9410$)$ between $K_{V_{\text {row }}}$ and the input variables. To illustrate the use of Equation (8), suppose we would like to determine the flow coefficient for the $60 \%$ VOP case with $15 \%$ inlet volume fraction at row 2 of quarter 3 of disc 6 (i.e. the middle disc), the input variables will be Row $=2$, Quarter $=3$, Disc $=6, \mathrm{VOP}=0.6$ and $\alpha_{\text {inlet }}=0.15$. Substituting these

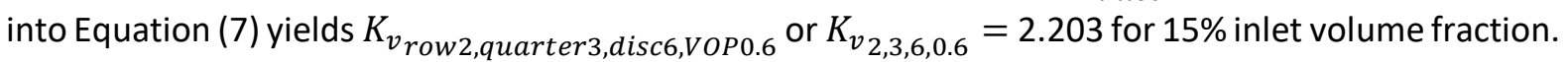
The correlation-calculated $K_{V_{\text {row }}}$ deviates from the CFD-obtained $K_{V_{\text {row }}}$ for that row location (i.e. 2.151 ) by $2.4 \%$. A second example: to determine the flow coefficient for the $100 \%$ VOP case with $10 \%$ inlet volume fraction at row 4 of quarter 3 of disc 1 (i.e. the top disc), the input variables will be Row $=4$, Quarter $=3$, Disc $=1, \mathrm{VOP}=1$ and $\alpha_{\text {inlet }}=0.10$. Substituting these into Equation (7) yields

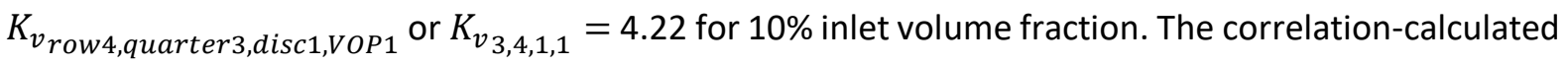
$K_{V_{\text {row }}}$ deviates from the CFD-obtained $K_{V_{\text {row }}}$ for that row location (i.e. 8.01) by $3.76 \%$. But as shown in Figure 16 (b), some predicted points can deviate by up to $20 \%$, giving an average deviation of $12 \%$ for the entire data set and can be considered acceptable. This demonstrates the equation's accuracy in determining the local flow coefficient of any disc row by entering information on its location within the trim and a value for the local air volume fraction at that disc row predicted by Equation (7). Furthermore, the accuracy given by Equation (7) is notwithstanding the amount of scatter the local volume fraction equation produced at lower locations in the trim where the air volume fraction is small or negligible. It hence gives us a model that is useful for designers in incorporating local $K_{v}$ information into design methodologies and this can lead to trim/valve designs that minimise the occurrence of problems such as cavitation and flashing.

\subsubsection{Cross-sectional phase non-uniformity: the distribution parameter $\boldsymbol{C}_{\boldsymbol{o}}$}

The distribution parameter $C_{o}$ is a constant that describes the effect of non-uniform flow. It indicates the difference in cross-sectional area averaged liquid and gas velocities as a result of the radial void distribution as well as of the phase velocity [35]. It was first defined for pipes by Zuber and Findlay [36] and we propose here to extend it to quantitatively describe the phase non-uniformities within the valve trim that were qualitatively shown in Sections 3.2 and 3.3 above. Hence, $C_{o}$ can be defined for each disc quarter within the trim as follows:

$$
C_{\text {quarter }}=\frac{\frac{1}{A} \int_{A=0}^{A} \alpha v_{\text {mix }} d A}{\left[\frac{1}{A} \int_{A=0}^{A} v_{\text {mix }} d A\right]\left[\frac{1}{A} \int_{A=0}^{A} \alpha d A\right]}
$$

where $v_{\text {mix }}=v_{s g}+v_{s l}$ is the local volumetric flux density of the mixture (also known as the mixture velocity); with $v_{s g}$ and $v_{s l}$ given as $v_{s g}=\alpha v_{g}$, and $v_{s l}=(1-\alpha) v_{l}$ with $v_{g}$ and $v_{l}$ being the actual velocities of the gas and liquid phases respectively. The volumetric flux densities $v_{s g}$ and $v_{s l}$ are also known as the superficial gas and liquid velocities and are defined as the velocity of the gas or liquid as 
if it flows alone in the domain. Since for the CFD data, local phase velocities and area averaged volume fractions were extracted at discrete planes $(A-F)$ within each quarter of a disc within the trim, we can rewrite Equation (9) in discrete form:

$$
C_{o_{\text {quarter }}}=\frac{\frac{1}{N_{\text {rows }}} \sum_{\text {row }=i}^{N_{\text {rows }}} \alpha_{i} v_{\text {mix }, i}}{\frac{1}{N_{\text {rows }}}\left[\sum_{\text {row }=i}^{N} v_{\text {mix }, i}\right] \frac{1}{N_{\text {rows }}} \sum_{\text {row }=i}^{N_{\text {rows }}} \alpha_{i}}
$$

4

where $N_{\text {rows }}=5$ is the number of cylindrical rows in the trim. Local air and water velocities and air volume fractions were extracted at planes $A-F$ were averaged such that row 1 values are average of planes $A$ and $B$, row 2 values are the average of planes $B$ and $C$, and so on. This gives characteristic void fraction/air or liquid velocity in that row which were then averaged for each quarter. Inspecting Equation (10) reveals that $C_{o_{\text {quarter }}}$ is likely to be very sensitive to the void fraction $\alpha_{i}$ since at very low $\alpha_{i}$ will produce large values of $C_{o_{\text {quarter }}}$.

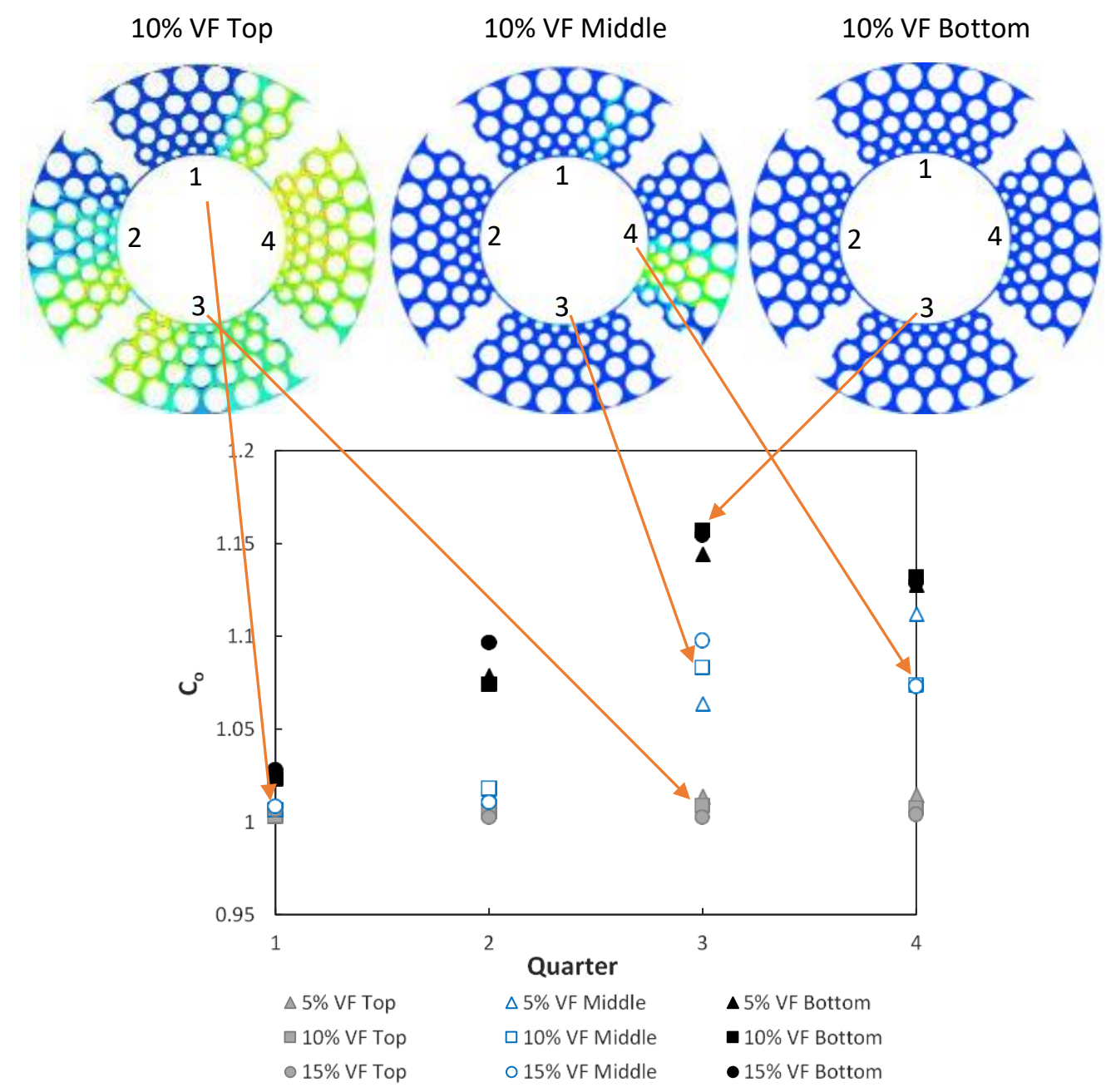

Figure 17: Distribution parameter characteristics at $100 \%$ valve opening

As a result, large $C_{o}$ values are more likely to occur at the bottom disc locations since there is phase stratification as we have previously shown, with air exiting at the upper locations of the trim. Figure 17 shows a plot of $C_{o}$ calculated using Equation (10) for each quarter at the disc locations considered for $5-15 \%$ inlet air volume fractions at $100 \%$ VOP. Above the plot is the void fraction distribution at $10 \%$ inlet volume fraction. It shows that $C_{o}$ values are spread between 1.007 and 1.158 . Quarters with 
low $C_{o}$ values (i.e. nearer to 1 ) denote air-rich quarters while those with values closer to 1.158 are indicative of water-rich quarters. Those with intermediate values denote quarters with the most nonuniformity in terms of phase distribution. It must be noted that, by its definition the distribution parameter gives the contribution of the phase fraction as well as volumetric flux density (or mixture velocity) in that quarter. Hence, a quarter with low air void fraction and high mixture velocity will have a similar $C_{o}$ value with one that has a high air void fraction and low volumetric flux density. A clear example is Quarter 3 of the middle disc at $10 \%$ inlet volume fraction. While the void fraction distribution shows that there is an almost negligible amount of air in that quarter, the extracted data shows that its velocity is rather high at 60 to $80 \%$ of the water phase. In effect, the distribution parameter tells us the relative contribution of the air phase in a quarter with respect to both its volume fraction as well as its velocity. Finally, the range of $C_{o}$ values obtained in this study is consistent with values reported by Kataoka and Ishii [35], Zuber and Findlay [36], and Hibiki and Ishii [37] in which $C_{o}$ varied between 1.0 and 1.5 for axisymmetric vertical pipe flows. Zuber and Findlay [36] noted that for fully established profiles (which is the case in this study as the flow analysis was carried out at steady state), the value of $C_{o}$ remains fairly constant at 1.0 for flat profiles, and up to 1.5 for peaked profiles. The values obtained for the current valve trim are within this range (i.e. 1.007 to 1.158) and consistent with the Zuber and Findlay's theory. Adopting a power law relationship as was previously done for $\alpha_{\text {row }}$ and $K_{v_{\text {row }}}$ in section 3.4.1, we correlate $C_{o_{\text {quarter }}}$ as a function of localised position in the trim, valve opening position and local air void fraction:

$$
C_{o_{\text {quarter }}}=\frac{0.93(\text { Quarter }+1)^{0.05}}{(V O P+1)^{-0.06}(\text { Disk }+1)^{0.06}} \alpha_{\text {quarter }}^{0.005}
$$

where $\alpha_{\text {quarter }}$ is calculated by averaging all $\alpha_{\text {row }}$ values in that quarter. Operatively, this is done using Equation (7) and averaging successive rows of air void fractions in that quarter to obtain $\alpha_{\text {quarter }}$ as follows: $\alpha_{\text {quarter }}=\frac{1}{N_{\text {rows }}} \sum_{i=1}^{N_{\text {rows }}} \alpha_{\text {row } i}$. The indices do not appear significant at first glance. However, since the range of $C_{o \text { quarter }}$ is narrow (between 1.0 and 1.158), a slight change in any of the input variables on the right-hand side produces a marked change in the distribution parameter.

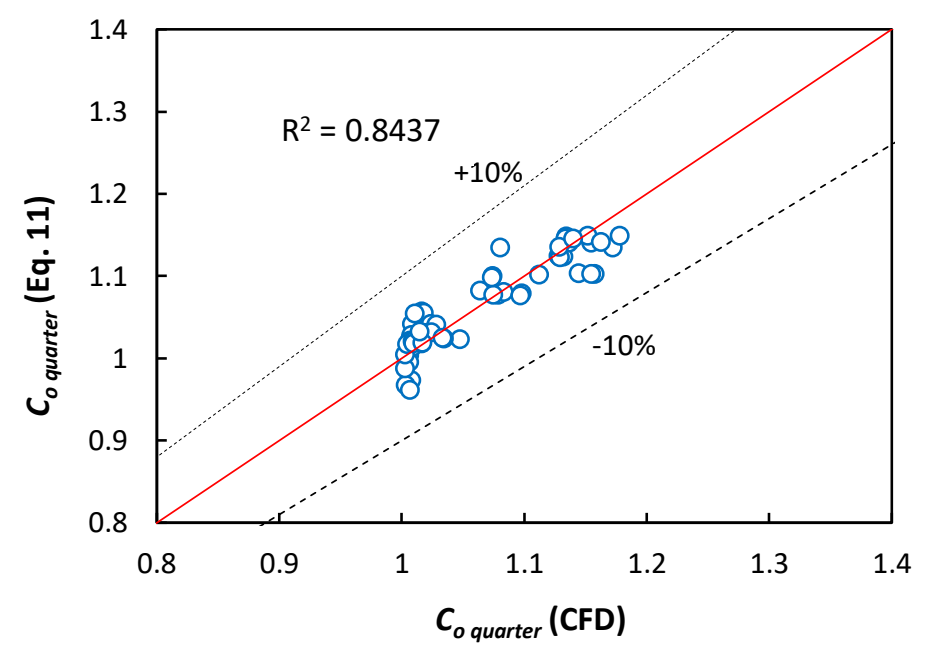

Figure 18: Comparison of $C_{o}$ obtained by Equation (11) and CFD data.

As shown in Figure 18, all the data points were predicted by the correlation to well within a $\pm 10 \%$ error band fairly evenly spread around the mean line giving an average absolute percentage error (AAPE) of $1.66 \%$ and $R^{2}$ value of 0.8437 ; suggesting a good match between Equation (11) and the data. Summarily, the detailed qualitative investigation of local $\alpha, K_{v}$, and $C_{o}$ presented here along with the 
relationships presented provide insight into the local flow behaviour in a multistage control valve trim with inter-stage recovery. The quantitative localised estimates provided by the correlations can be integrated in global parameter-based design procedures for severe-service control valves by integrating $\alpha, K_{v}$, and $C_{o}$ for better quality assurance in the valves.

\section{Conclusions}

6

In this study, we have presented a well-validated CFD study to investigate the local flow characteristics in a severe-service control valve trim under multiphase gas and liquid flow. Three different air inlet volume fractions $(5,10$, and $15 \%)$ were tested at $60 \%$ and $100 \%$ valve opening positions. To validate the CFD model, experiments were carried out and the global pressure drop across the valve between the CFD and experiments were within $\pm 5 \%$ of each other. The results from the CFD simulations showed phase stratification with the air exiting the trim towards the top for both valve opening conditions investigated. Uniform pressure distribution was obtained azimuthally, and the local pressures progressively decrease through the flow paths between the staggered cylinder rows as the flow moves from the inlet region at the trim circumference to the outlet at the centre of trim. Pressure drop increases from the top to the bottom of the trim with increase in inlet air volume fraction. However, the local water/air velocity and air void fraction distributions were not as axisymmetric. The water velocity was more uniformly distributed than that of the air. It was observed that water displaces the air phase at the vicinity of the entrance pipe and the highest air volume fractions occurred near the top and at locations opposite the inlet pipe. In order to quantify the phase non-uniformities observed, data extracted from specified planes were used to obtain relationships for the local void fraction distribution at rows in each disc quarter. This in turn was used to calculate the flow coefficient $K_{v_{\text {row }}}$ and the results show excellent agreement between the data and the correlation as most points were within a $\pm 20 \%$ error band. Furthermore, a correlation was generated for the distribution parameter $C_{o}$ for each disc quarter in the trim such that its value lies between 1.007 and 1.158 for the three inlet volume fractions and valve openings tested. The highest $C_{o}$ values, i.e. those closer to 1.158 are indicative of a water-dominated disc quarter. Conversely, a value closer to 1.007 indicates an air-rich quarter. In conclusion, the detailed investigation we presented permits local multiphase flow characteristics to be established and embedded into the design methodologies of severe-service control valves.

\section{Declaration of interest}

None declared.

\section{CRediT statement}

D. Singh: Software, Data Curation, Experimentation, Analysis, Writing - original draft, review and editing. A. M. Aliyu: Writing - original draft, review and editing, Data Curation, Analysis. M. Charlton: Writing - review and editing R. Mishra: Writing - original draft, review and editing, Analysis, Supervision. T. Asim: Software. A. C. Oliveira: Experimentation.

\section{References}

[1] S.-K. S.-H. Kang, J.-Y. Yoon, S.-K. S.-H. Kang, and B.-H. Lee, "Numerical and experimental investigation on backward fitting effect on valve flow coefficient," Proc. Inst. Mech. Eng. Part E J. Process Mech. Eng., vol. 220, no. 4, pp. 217-220, Nov. 2006.

[2] Q. Yang, Z. Zhang, M. Liu, and J. Hu, "Numerical Simulation of Fluid Flow inside the Valve," 
Procedia Eng., vol. 23, pp. 543-550, 2011.

[3] V. V. Yagov and M. V. Minko, "Simulating droplet entrainment in adiabatic disperse-annular two-phase flows," Therm. Eng., vol. 60, no. 7, pp. 521-526, 2013.

[4] F. Lin and G. A. Schohl, "CFD Prediction and Validation of Butterfly Valve Hydrodynamic Forces," in Critical Transitions in Water and Environmental Resources Management, 2004, pp. 1-8.

[5] Y. J. An, B. J. Kim, and B. R. Shin, "Numerical analysis of 3-D flow through LNG marine control valves for their advanced design," J. Mech. Sci. Technol., vol. 22, no. 10, pp. 1998-2005, Oct. 2008.

[6] E. Lisowski and J. Rajda, "CFD analysis of pressure loss during flow by hydraulic directional control valve constructed from logic valves," Energy Convers. Manag., vol. 65, pp. 285-291, Jan. 2013.

[7] E. Lisowski, W. Czyżycki, and J. Rajda, "Multifunctional four-port directional control valve constructed from logic valves," Energy Convers. Manag., vol. 87, pp. 905-913, Nov. 2014.

[8] E. Lisowski and G. Filo, "CFD analysis of the characteristics of a proportional flow control valve with an innovative opening shape," Energy Convers. Manag., vol. 123, pp. 15-28, Sep. 2016.

[9] T. Asim, M. Charlton, and R. Mishra, "CFD based investigations for the design of severe service control valves used in energy systems," Energy Convers. Manag., vol. 153, pp. 288-303, Dec. 2017.

[10] T. Asim, R. Mishra, A. Oliveira, and M. Charlton, "Effects of the geometrical features of flow paths on the flow capacity of a control valve trim," J. Pet. Sci. Eng., vol. 172, pp. 124-138, Jan. 2019.

[11] T. Asim, A. Oliveira, M. Charlton, and R. Mishra, "Improved design of a multi-stage continuousresistance trim for minimum energy loss in control valves," Energy, vol. 174, pp. 954-971, May 2019.

[12] BS EN 60534-2-1, "Industrial-process control valves. Part 2-1: Flow capacity - Sizing equations for fluid flow under installed conditions." 2011.

[13] R. Diener and J. Schmidt, "Sizing of throttling device for gas/liquid two-phase flow part 2: Control valves, orifices, and nozzles," Process Saf. Prog., vol. 24, no. 1, pp. 29-37, Mar. 2005.

[14] W. Dempster and M. Alshaikh, "An Investigation of the Two Phase Flow and Force Characteristics of a Safety Valve," Procedia Eng., vol. 130, pp. 77-86, Jan. 2015.

[15] W. Dempster and M. Alshaikh, "CFD prediction of safety valve disc forces under two phase flow conditions," Am. Soc. Mech. Eng. Press. Vessel. Pip. Div. PVP, vol. 3A, pp. 1-7, 2018.

[16] BS EN 60534-2-3, "Industrial-process control valves. Part 2-3: Flow capacity - Test procedures." 1998.

[17] R. Diener, L. Friedel, and J. Kiesbauer, "Auslegung von Stellgeräten bei Zweiphasenströmung (Sizing control valves for two-phase flow)," Autom. Prax., vol. 42, no. 3, 2001.

[18] R. Diener, J. Kiesbauer, and J. Schmidt, "Improved control valve sizing for multiphase flow," Hydrocarb. Process., vol. 84, no. 3, pp. 59-64, 2005.

[19] C. W. Sheldon and C. B. Schuder, "Sizing Control Valves for Liquid-Gas Mixtures," Instruments Control Syst., vol. 38, 1965. 
[20] R. Diener and J. Schmidt, "Sizing of throttling device for gas/liquid two-phase flow part 1: Safety valves," Process Saf. Prog., vol. 23, no. 4, pp. 335-344, 2004.

[21] J. C. Leung, "A generalized correlation for one-component homogeneous equilibrium flashing choked flow," AIChE J., vol. 32, no. 10, pp. 1743-1746, Oct. 1986.

[22] J. C. Leung, "Similarity between flashing and nonflashing two-phase flows," AIChE J., vol. 36, no. 5, pp. 797-800, May 1990.

[23] R. Diener, "Berechnung und Messung der Massendurchsatzcharacteristik von Stellventilen bei Zweiphasenströmung (Experimental and calculated control valve two-phase mass flow characteristic)," 2000.

[24] BS EN 60534-2-5, "Industrial-process control valves. Part 2-5: Flow Capacity - Sizing equations for fluid flow through multistage control valves with interstage recovery." 2003.

[25] J. H. Ferziger and M. Peric, Computational Methods for Fluid Dynamics, 3rd ed. Berlin: Springer, 2002.

[26] C. Pozrikidis, Fluid Dynamics: Theory, Computation, and Numerical Simulation. Boston, MA: Springer US, 2017.

[27] H. K. Versteeg and W. Malalasekera, An Introduction to Computational Fluid Dynamics: The Finite Volume Method, 2nd ed. Harlow, UK: Pearson Education, 2007.

[28] T. Cebeci, J. P. Shao, F. Kafyeke, and E. Laurendeau, Computational Fluid Dynamics for Engineers. Berlin, Heidelberg: Springer Berlin Heidelberg, 2005.

[29] J. Blazek, Computational Fluid Dynamics: Principles and Applications, 3rd ed. Amsterdam: Elsevier, 2015.

[30] J. A. Schetz and A. E. Fuhs, Fundamentals of fluid mechanics. John Wiley \& Sons, 1999.

[31] F. R. Menter, "Two-equation eddy-viscosity turbulence models for engineering applications," AIAA J., vol. 32, no. 8, pp. 1598-1605, Aug. 1994.

[32] T. Asim and R. Mishra, "Optimal design of hydraulic capsule pipelines transporting spherical capsules," Can. J. Chem. Eng., vol. 94, no. 5, pp. 966-979, May 2016.

[33] S. Pour, S., K. Mohanarangam, S. Vahaji, S. C. P. Cheung, and J. Tu, Visualisation of gas-liquid bubbly flows in a large diameter pipe with $90^{\circ}$ bend. I Vis 21, 585-596 (2018). https://doi.org/10.1007/s12650-018-0486-2

[34] Aliyu, A.M., Seo, H., Kim, M. et al. An experimental study on the characteristics of ejectorgenerated bubble swarms. J Vis 21, 711-728 (2018). https://doi.org/10.1007/s12650-0180494-2

[35] I. Kataoka and M. Ishii, "Drift flux model for large diameter pipe and new correlation for pool void fraction," Int. J. Heat Mass Transf., vol. 30, no. 9, pp. 1927-1939, Sep. 1987.

[36] N. Zuber and J. A. Findlay, "Average Volumetric Concentration in Two-Phase Flow Systems," J. Heat Transfer, vol. 87, no. 4, pp. 453-468, Nov. 1965.

[37] T. Hibiki and M. Ishii, "One-dimensional drift - flux model for two-phase flow in a large diameter pipe," Int. J. Heat Mass Transf., vol. 46, pp. 1773-1790, 2003. 


\section{Appendix: Supplementary figures}

Inlet vol.

fraction

Top disc

Middle disc

Bottom disc

[\%]

5
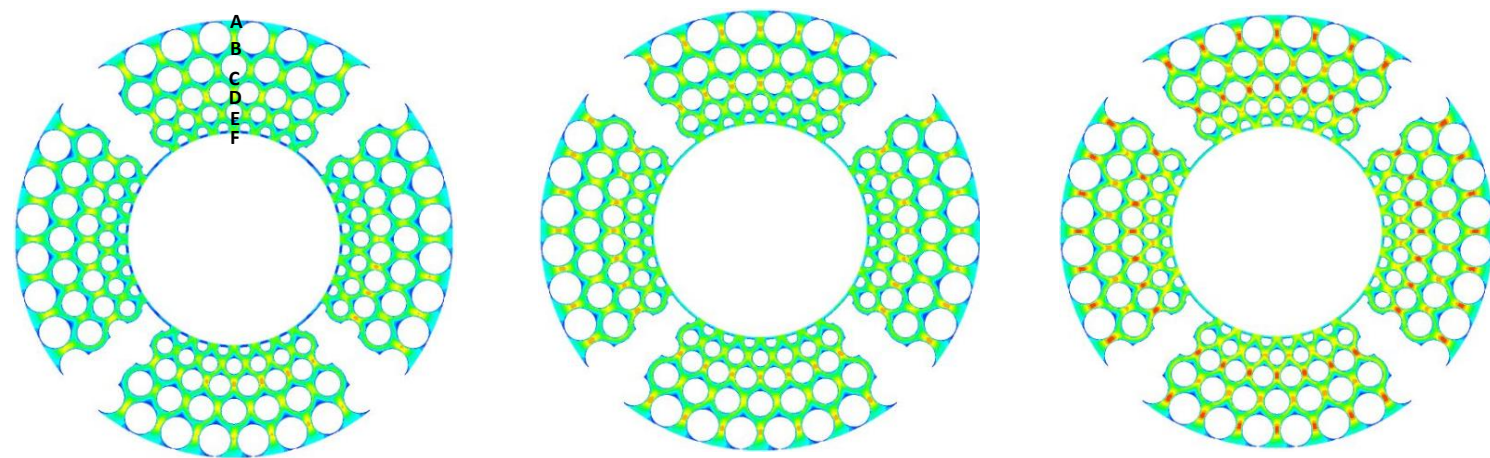

10
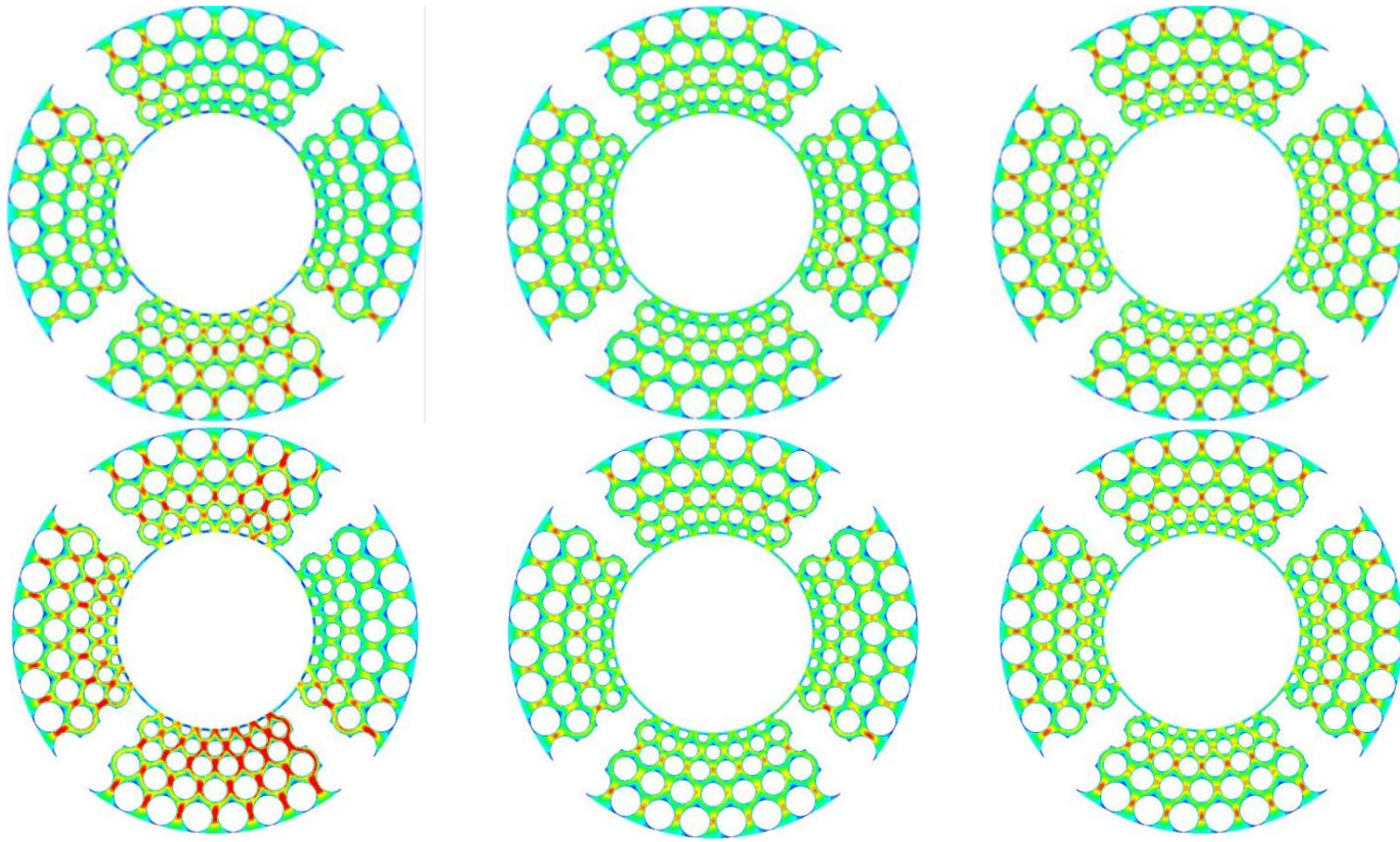

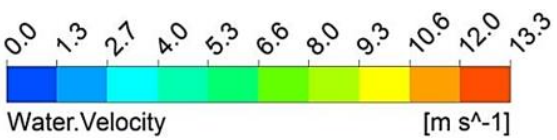

Figure A1: Water velocity distribution at the top, middle and bottom discs, at 5, 10, and 15\% air inlet volume fractions respectively and at $100 \%$ valve opening (average inlet velocity of $10 \mathrm{~m} / \mathrm{s}$ ). 
Inlet vol. fraction [\%]

5

10
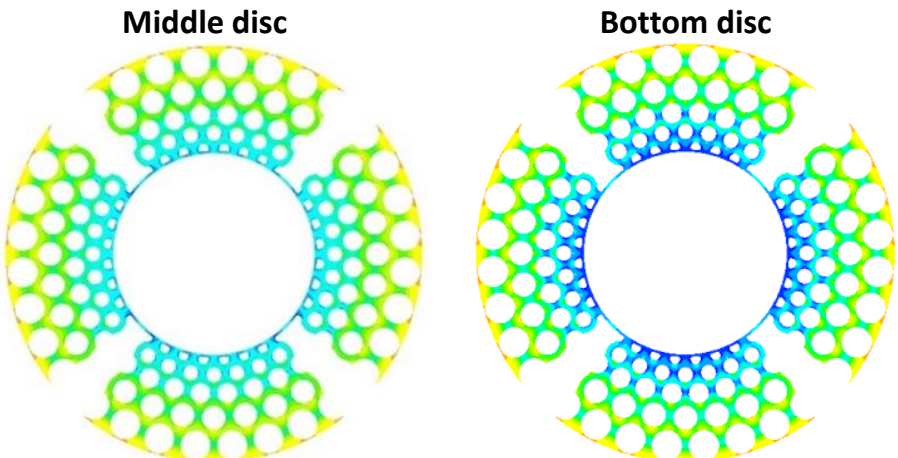

15
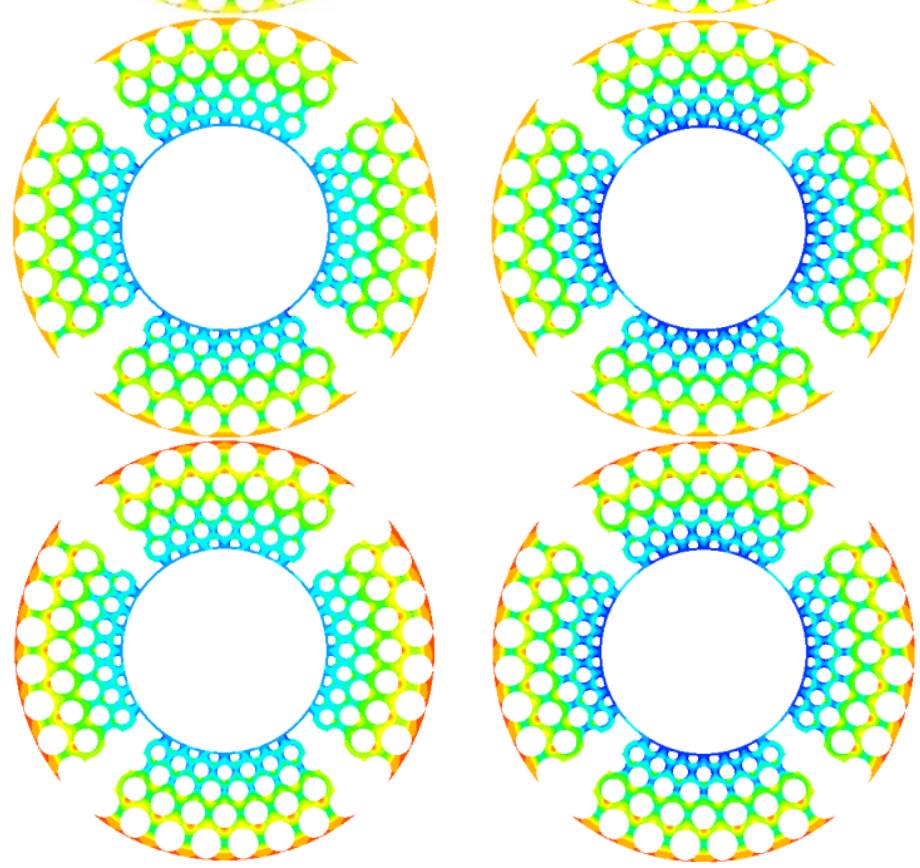

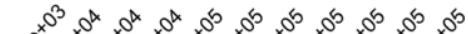

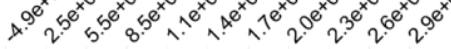

Pressure

$[\mathrm{Pa}]$

Figure A2: Pressure distributions at the middle disc for (a) $5 \%$, (b) $10 \%$, and (c) $15 \%$ inlet air volume fraction at $60 \%$ valve opening position (average inlet velocity of $10 \mathrm{~m} / \mathrm{s}$ ) 


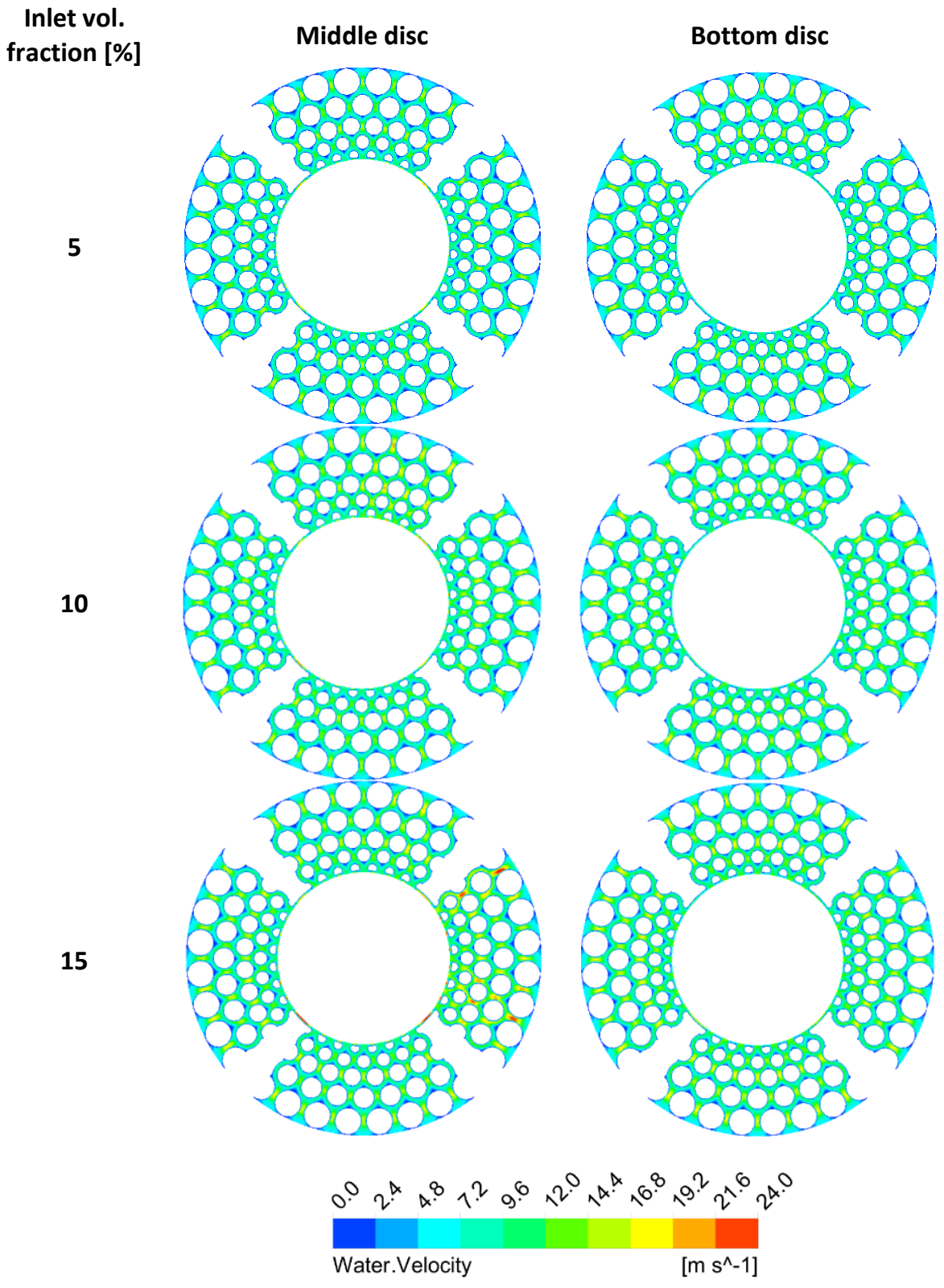

Figure A3: Water velocity distribution in the middle and bottom discs, at 5, 10, and $15 \%$ air inlet volume fractions respectively and at $60 \%$ VOP (average inlet velocity of $10 \mathrm{~m} / \mathrm{s}$ ). 